\title{
Derivation and Comparative Assessment of Retinal Pigment Epithelium from Human Embryonic Stem Cells Using Transcriptomics
}

\author{
IRINA KLIMANSKAYA, ${ }^{1}$ JASON HIPP ${ }^{2}$ KOUROUS A. REZAI, ${ }^{3}$ MICHAEL WEST, ${ }^{1}$ \\ ANTHONY ATALA, ${ }^{2}$ and ROBERT LANZA ${ }^{1,2}$
}

\begin{abstract}
Human stem-cell derivatives are likely to play an important role in the future of regenerative medicine. Evaluation and comparison to their in vivo counterparts is critical for assessment of their therapeutic potential. Transcriptomics was used to compare a new differentiation derivative of human embryonic stem (hES) cells - retinal pigment epithelium (RPE) - to human fetal RPE. Several hES cell lines were differentiated into putative RPE, which expressed RPEspecific molecular markers and was capable of phagocytosis, an important RPE function. Isolated hES cell-derived RPE was able to transdifferentiate into cells of neuronal lineage and redifferentiate into RPE-like cells through multiple passages ( $>30$ Population doublings). Gene expression profiling demonstrated their higher similarity to primary RPE tissue than of existing human RPE cell lines D407 and ARPE-19, which has been shown to attenuate loss of visual function in animals. This is the first report of the isolation and characterization of putative RPE cells from hES cells, as well as the first application of transcriptomics to assess embryonic stem-cell derivatives and their in vivo counterparts-a "differentiomics" outlook. We describe for the first time, a differentiation system that does not require coculture with animal cells or factors, thus allowing the production of zoonoses-free RPE cells suitable for subretinal transplantation in patients with retinal degenerative diseases. With the further development of therapeutic cloning, or the creation of the banks of homozygous human leucocyte antigen (HLA) hES cells using parthenogenesis, RPE lines could be generated to overcome the problem of immune rejection and could be one of the nearest term applications of stem-cell technology.
\end{abstract}

\section{INTRODUCTION}

G ENE EXPRESSION PROFILING ALlOWS the analysis of thousands of transcripts within the cell. To date, the primary application of this technology to stem-cell research has been the discovery of potential "stemness" genes in embryonic stem (ES) cells and their downregulation in dif- ferentiated cells (Abeyta et al., 2004; Ivanova et al., 2002; Ramalho-Santos et al., 2002; Sato et al., 2003). These studies were carried out with differentiating ES cells' cultures comprised of their various differentiation derivatives, thus limiting the possibility of interpretation of differentiationassociated genes' data sets. We have isolated a novel differentiation derivative of human em-

${ }^{1}$ Advanced Cell Technology, Worcester, Massachusetts.

${ }^{2}$ Institute for Regenerative Medicine, Wake Forest University School of Medicine, Winston-Salem, North Carolina.

${ }^{3}$ Department of Ophthalmology and Visual Science, University of Chicago, Chicago, Illinois. 
bryonic stem (hES) cells, putative retinal pigment epithelium, a specialized eye tissue involved in photoreceptor maintenance and whose dysfunction can lead to photoreceptor deterioration and blindness. Transcriptomics was used for the first time as an approach to evaluate this in vitro EScell derivative to its in vivo counterpart.

Retinal pigment epithelium (RPE) is a neuroectodermal derivative essential for the survival of photoreceptors. This densely pigmented epithelial monolayer is located between the choroid and neural retina and serves as a part of a barrier between the bloodstream and retina. Its functions include phagocytosis of shed rod and cone outer segments, absorption of stray light, vitamin A metabolism, regeneration of retinoids, and tissue repair (Fisher and Reh, 2001; Grierson et al., 1994; Marmorstein et al., 1998). There are several known molecular markers of the RPE, including cellular retinaldehyde-binding protein (CRALBP), a cytoplasmic protein also found in apical microvilli (Bunt-Milam and Saari, 1983); RPE65, a cytoplasmic protein involved in retinoid metabolism (Ma et al., 2001; Redmond et al., 1998); bestrophin, the product of the Best vitelliform macular dystrophy gene (VMD2; Marmorstein et al., 2000), and pigment epithelium derived factor (PEDF) a 48kD secreted protein with angiostatic properties (Jablonski et al., 2000, Karakousis et al., 2001).

An unusual feature of the RPE is its apparent plasticity. RPE cells are normally mitotically quiescent, but can begin to divide in response to injury or photocoagulation. RPE cells adjacent to the injury flatten and proliferate forming a new monolayer (Zhao et al., 1997). Several studies have indicated that the RPE monolayer can produce cells of fibroblast appearance that can later revert to their original RPE morphology (Grierson et al., 1994, Kirchhof et al., 1988, Lee et al., 2001). In vitro, depending on the combination of growth factors and substratum, RPE can be maintained as an epithelium or rapidly dedifferentiate and proliferate (Opas and Dziak, 1994; Zhao et al., 1997). Interestingly, the epithelial phenotype can be reestablished in long-term quiescent cultures (Grierson et al., 1994).

In mammalian development, RPE shares the same progenitor with neural retina, the neuroepithelium of the optic vesicle. Under certain conditions, it has been suggested that RPE can transdifferentiate into neuronal progenitors (Opas and
Dziak, 1994), neurons (Chen et al., 2003, Vinores et al., 1995), and lens epithelium (Eguchi, 1986). One of the factors that can stimulate the change of RPE into neurons is bFGF (Opaz and Dziak, 1994), and this is associated with the expression of transcriptional activators normally required for the eye development, including $\mathrm{rx} / \mathrm{rax}$, chx10/ vsx-2/alx, ots-1, otx-2, six3/optx, six6/optx2, mitf, and pax6/pax2 (Baumer et al., 2003; Fischer and Reh, 2001). Recently, it has been shown that the margins of the chick retina contain neural stem cells (Fischer and Reh, 2000) and that the pigmented cells in that area expressing pax6/mitf can form neuronal cells in response to FGF (Fisher and Reh, 2001).

The degeneration of RPE with age is thought to play a critical role in the pathogenesis of agerelated macular degeneration (ARMD). Although different approaches have been proposed for the treatment of ARMD, none of them have proved to be successful in the treatment of this devastating disease. Animal studies indicate that degenerated RPE cells can be replaced successfully by transplanting donor RPE cells, rescuing the host photoreceptors, and attenuating loss of visual function (Coffey et al., 2002; Lund et al., 2001). Pigmented epithelial cells have been derived from ES cells of the Cynomologous monkey and they provided similar protection when transplanted to the subretinal space of rats (Haruta et al., 2004).

This study reports, for the first time, the isolation of putative RPE cells from several spontaneously differentiating human ES cell lines and comparative transcriptomic assessment of ES cell derivatives versus their in vivo counterparts.

\section{MATERIALS AND METHODS}

\section{hES cell lines}

The hES cell lines used in this study were the previously described H1, H7, and H9 (Thomson et al., 1998; National Institutes of Health-registered as WA01, WA07, and WA09); six new lines derived with the use of private funds (Cowan et al., 2004); and two newly derived and partially characterized lines of human inner cell mass-derived ES-like cells (the lines are still undergoing characterization). Human frozen blastocysts or cleaved embryos were donated to the study, ap- 
proved by two institutional review boards, by couples who had completed their fertility treatments. hES cells were maintained on mitomycin $\mathrm{C}$-treated mouse embryonic fibroblasts (MEFs) in growth medium: knockout high glucose DMEM supplemented with $500 \mathrm{u} / \mathrm{mL}$ of penicillin, 500 $\mathrm{ug} / \mathrm{mL}$ of streptomycin, $1 \%$ nonessential amino acids solution, $2 \mathrm{mM}$ of GlutaMAX-I, Carlsbad, CA $0.1 \mathrm{mM} \beta$-mercaptoethanol, $4 \mathrm{ng} / \mathrm{mL}$ bFGF (Invitrogen, Carlsbad, CA), $10 \mathrm{ng} / \mathrm{mL}$ human LIF (Chemicon, Temecula, CA), $8 \%$ of Serum Replacement (SR; Invitrogen) and $8 \%$ Plasmanate (Bayer Research Triangle Park, NC). The cells were routinely passaged with trypsin at a ratio of 1:3-1:6 every 3-5 days (for detailed procedures see Klimanskaya and McMahon, 2004).

Differentiation experiments were performed with adherent hES cells grown on MEFs, or feeder-free, or with embryoid bodies (EBs). For adherent differentiation, hES cells were allowed to overgrow on MEFs until the hES colonies lost their tight borders and became multilayered, at which time the culture media was replaced with an EB medium: this was the same as the growth medium except it did not contain bFGF, LIF, and Plasmanate; the SR concentration was 13\% (usually $8-10$ days after passaging). The medium was changed every 1-2 days. For EB formation, hES cells were trypsinized and cultured in EB medium on Costar brand low adherence plates.

\section{Immunostaining}

Cells were fixed with $2 \%$ paraformaldehyde, permeabilized with $0.1 \%$ NP-40 for localization of intracellular antigens, and blocked with 10\% goat serum, and 10\% donkey serum (Jackson Immunoresearch Laboratories, West Grove, PA) in phosphate buffered saline (PBS) (Invitrogen) for at least 1 hour. Incubation with primary antibodies was carried out overnight at $4^{\circ} \mathrm{C}$ and the fluorescently labeled secondary antibodies (Jackson Immunoresearch Laboratories) were added for 1 hour. Between all incubations, specimens were washed with $0.1 \%$ Tween-20 (Sigma, St. Louis, MO) in PBS 3-5 times, 10-15 minutes each wash. Specimens were mounted using Vectashield with DAPI (Vector Laboratories, Burlingame, CA) and observed under fluorescent microscope (Nikon global headquarters Kawasaki, Kanagawa, Japan). Antibodies used were antipax6, anti-tubulin $\beta$ III from Covance (Berkeley,
CA), and anti-bestrophin from Novus Biologicals (Littleton, CO); the anti-CRALBP antibody was a generous gift from Dr. John Saari, University of Washington, Seattle, WA.

\section{Isolation and passaging of RPE-like cells}

Adherent cultures of hES cells were rinsed with PBS twice and incubated in $0.25 \%$ Trypsin $/ 1 \mathrm{mM}$ of ethylenediaminetetraacetic acid (EDTA) (Invitrogen) at $37^{\circ} \mathrm{C}$ until the monolayer loosened. Cells from the pigmented regions were scraped off with a glass capillary, transferred to an MEF medium, centrifuged at $160 \times \mathrm{g}$, and plated onto gelatin-coated plates in RPE medium (knockout high glucose DMEM supplemented with 500 $\mathrm{u} / \mathrm{mL}$ of penicillin, $500 \mathrm{ug} / \mathrm{mL}$ of streptomycin, $1 \%$ nonessential aminoacids solution, $2 \mathrm{mM}$ of GlutaMAX I, $0.1 \mathrm{mM} \beta$-mercaptoethanol, 7\% SR, and $5 \%$ fetal bovine serum [FBS]). The medium was changed after the cells attached (usually in 1-2 days) and every 5-7 days after that; the cells were passaged every 2-4 weeks with $0.05 \%$ Trypsin/0.53 mM of EDTA (Invitrogen).

\section{Western blot and enzyme-linked immunosorbent assay}

Samples were prepared in Laemmli buffer (Laemmli, 1970) , supplemented with a $5 \% \beta$-mercaptoethanol and protease inhibitor cocktail (Roche, Nutley, NJ), boiled for 5 minutes and loaded onto a $8 \%-16 \%$ gradient gel (Bio-Rad, Hercules, CA) using a Mini-Protean apparatus; the gels were run at 25-30 mA per gel; proteins were transferred to a 0.2 Nitrocellulose membrane (Schleicher and Shull, Keene, NH) at 20 volts overnight. Blots were briefly stained with Ponceau Red (Sigma) to visualize the bands, washed with Milli-Q water (Millipore, Bedford, MA), and blocked for 1 hour with 5\% nonfat dry milk in $0.1 \%$ Tris buffered saline TBST (Bio-Rad) were added for 2 hours followed by three 15-minute washes with TBST; peroxidase-conjugated secondary antibodies were added for 1 hour and the washes were repeated. Blots were detected using an ECL system with Super-Signal reagent (Pierce, Iselin, NJ). A PEDF enzyme-linked immunosorbent assay (ELISA) was performed on cell lysates using a PEDF ELISA kit (Chemicon) according to the manufacturer's protocol. 


\section{Real-time RT-PCR}

Total ribonucleic acid (RNA) was purified from differentiating ES cultures by a two-step procedure. Crude RNA was isolated using Trizol reagent (Invitrogen) and further purified on RNeazy minicolumns (Qiagen, Valencia, CA). The levels of RPE65 transcripts were monitored by real-time polymerase chain reaction using a commercial primer set for RPE65 detection (Assay on Demand \# Hs00165642, Applied Biosystems) and Quantitect Probe RT [reaction time]-PCR reagents (Qiagen), according to the manufacturer's protocol.

RNA isolation and hybridization to Human Affymetrix GeneChip ${ }^{\circledR}$ U133 Plus 2.0 Set (Affymetrix, Santa Clara, CA)

Total RNA isolations, Affymetrix array hybridization, and raw data collection was performed using standard protocols at Genome Explorations (Memphis, TN). Each RNA sample was checked for quality control by an Agilent Bioanalizer 2100 (Palo Alto, CA). Chips were read by the Affymetrix GCS 3000 scanner.

\section{Phagocytosis assay and electron microscopy}

hES-RPE cells were grown on gelatin-coated 6well plates until the majority of the cells looked fully differentiated (pigmented epithelial appearance), incubated with $10^{8}$ beads/mL suspension of latex beads (Sigma) for up to 24 hours, fixed in $2.5 \%$ glutaraldehyde in PBS for 30 minutes, rinsed with PBS, and were postfixed with $1 \%$ osmium tetroxide. Subsequently, the cells were washed and dehydrated through a graded series of alcohols and embedded in epoxy resin. Thin sections of the samples embedded in epoxy resin were double-stained with lead citrate and uranyl acetate and then observed at $80 \mathrm{keV}$ in a Phillips (Global headquarters Eindhoven, The Netherlands) transmission electron microscopy. Phagocytosis of Fluorescein isothiocyanate-labeled rod outer segments was performed using flow cytometry, as described by Kennedy and coauthors (Kennedy et al., 1996).

Total RNA isolations, Affymetrix array hybridization, and raw data collection was performed using standard protocols at Genome Explorations (Memphis, TN). Each RNA sample was checked for quality control by the Agilent
Bioanalizer 2100 (Palo Alto, CA). Chips were read by the Affymetrix GCS 3000 scanner. Microarrays were performed using the Affymetrix U133 Plus 2.0 GeneChip on human embryonic stem cell (SC)-derived retinal pigmented epithelium (hESRPE) and those that have transdifferentiated into neural precursors (TD), by pooling RNA from multiple wells to minimize biologic variability and noise. Genes were then filtered based on their present detection call ( $p$ value of $<0.04$ ) using the Affymetrix Microarray Suite (MAS) Version 5.0, and converted to their Locus Link ID, which identified 8888 well-annotated genes as present in hES-RPE, with 7165 in TD.

\section{U133 Plus 2.0 chip analysis}

Raw data from the hybridization experiments were processed using the MAS 5.0. The readings from each of the arrays were globally scaled to yield the same target overall array intensity and the scaling factors thus generated were checked against each other for consistency between chips. Transcript detection calls and signal intensities for the 54,675 probe sets of each U133 Plus 2.0 array were extracted using the MAS 5.0, one-step Tukeybiweight algorithm. Raw Affymetrix data will be available at the Wake Forest Institute of Regenerative Medicine Web site, www.wfirm.org.

\section{RESULTS}

\section{Differentiation of hES cells and isolation of pigmented epithelium}

When hES cell cultures were allowed to overgrow and spontaneously differentiate, the majority of the early differentiating cells appeared neuronal, as evidenced by immunostaining with antibodies to pax6 and tubulin $\beta$ III. The colonies lost their typical undifferentiated morphology and formed three-dimensional multicellular structures. Within 2-3 weeks, after switching to a differentiation medium, clusters of polygonalshaped cells resembling columnar epithelium, surrounded by cells of neuronal origin (pax6 and tubulin $\beta$ III-positive, Fig. 1 A-D) were observed as well as other unidentified cell types. Over time, granules of brown pigment appeared in the cytoplasm of epithelial-like cells, and, in 6-8 weeks, well-defined clusters of polygonal pigmented cells coexisted in cultures with cells of other 
phenotypes (Fig. 1 E-G). The most densely pigmented cells were invariably located in the middle of the clusters, and as the cultures "matured," this dense pigmentation spread to the periphery. A similar phenomenon has been observed when cultured retina cells transdifferentiate into retinal pigment epithelium (Opas et al., 2001). Only a small fraction of hES cells in each culture produced pigmented cells over the course of $4-8$ weeks; such clusters were visible as "freckles" in the culture dishes (Fig. 1, E). In cultures of differentiating EBs, less than 1\% of EBs developed pigmented islands in the first 4-8 weeks (Fig. 1, $\mathrm{F}, \mathrm{H})$ whereas, over the course of 6-9 months, the cells on the surface of all EBs became pigmented.

Pigmented cells were isolated by either handpicking (as described in Materials and Methods) or by plating pigmented EBs without dissociation onto gelatin for outgrowth. The cells lost pigmentation and epithelial morphology as they divided and migrated away from the initial attachment site (Fig. 2, A, B). However, once confluency was established, the cells reverted to epithelial morphology and reexpressed pigment (Fig. 2, C,D) as has been previously described for RPE (Grisanti and Guidry, 1995; Opas and Dziak, 1994; Zhao et al., 1997). The pigmented epithelial cells were often organized as islands, surrounded by a small number of elongated, nonpigmented cells. These established monolayers of RPE-like cells were routinely passaged every 2-4 weeks and have undergone multiple passages (to date, up to 9).

\section{Assessment of hES cell-derived putative RPE}

Phagocytosis is an important function of RPE in the eye and plays a key role in the maintenance of photoreceptor function. We confirmed the ability of putative RPE to perform this function using a latex-bead assay as previously described (Haruta et al., 2004) and a rod outer segments (ROS) phagocytosis assay. Phagosomes formed around the latex beads and were detected inside the cells using transmission electron microscopy or TEM (Fig. 3A), indicating that they were capable of phagocytosis. Phagocytosis of FITClabeled ROS, as assessed by flow cytometry showed that $90 \%$ of the population of putative RPE cells were capable of RPE-specific phagocytosis (data not shown).

There are several characteristic RPE proteins, such as bestrophin, RPE65, CRALBP, and PEDF
(Karakousis et al., 2001; Ma et al., 2001; Marmorstein et al., 2000; Redmond et al., 1998), which were expressed in putative RPE cells. Western blot analysis confirmed the expression of CRALBP, PEDF, and bestrophin in these cells; PEDF secretion was also detected by ELISA in the conditioned medium and whole-cell lysates (not shown). The pattern of immunofluorescence localization of bestrophin and CRALBP correlated with the epithelial morphology of the cells and the level of pigmentation (Fig. 3, C-F, H). Real time RT-PCR confirmed expression of RPE65 in all hES-RPE samples analyzed (Fig. 3). Interestingly, mature cultures (7 weeks after passaging) had four- to ninefold more RPE65 mRNA than the control undifferentiated hES cells, whereas earlier passage (2-week) cultures only exceeded the control 1.5-2.5 fold (Fig. 3G).

\section{Comparative evaluation of}

$h E S-R P E$ by transcriptomics

hES-cell derivatives are likely to play an important role in the future of regenerative medicine. Qualitative assessment of these and other SC derivatives remains a challenge that could be approached using functional genomics. To test this, we analyzed the transcriptional profile of hES-RPE versus its in vivo counterpart, fetal RPE (feRPE) which has been extensively researched for its transplantation value. Both profiles were then compared with the previously published (Rogojina et al., 2003) transcriptomics data on human RPE cell lines ARPE-19 and D407.

The gene expression profile of our data set was compared to two human RPE cell lines (nontransformed ARPE-19 and transformed D407, Rogojina et al., 2003) to determine whether hES-RPE have similar global transcriptional profiles. To account for common housekeeping genes expressed in all cells, we used publicly available Affymetrix data sets from undifferentiated hES cells (H1 line, h1-hES; Sato et al., 2003) and bronchial epithelial cells (BE; Wright et al., 2004) as a control, based on its common epithelial origin that would allow to exclude common housekeeping and epithelial genes and identify RPE-specific genes.

Venn diagrams based on present calls (Fig. 4) illustrate the similarities and differences among hES-RPE, hES-RPE-TD, ARPE-19, D407, and feRPE. This similarity was further demonstrated by ignoring the genes expressed in all 3 cell types 

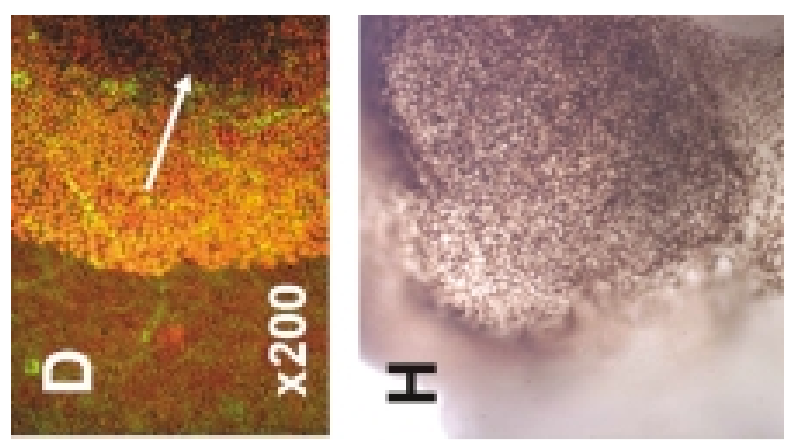

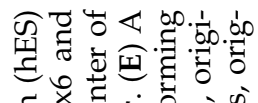

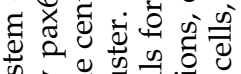
क 可

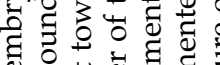

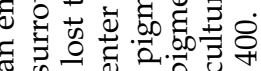

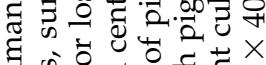

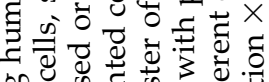
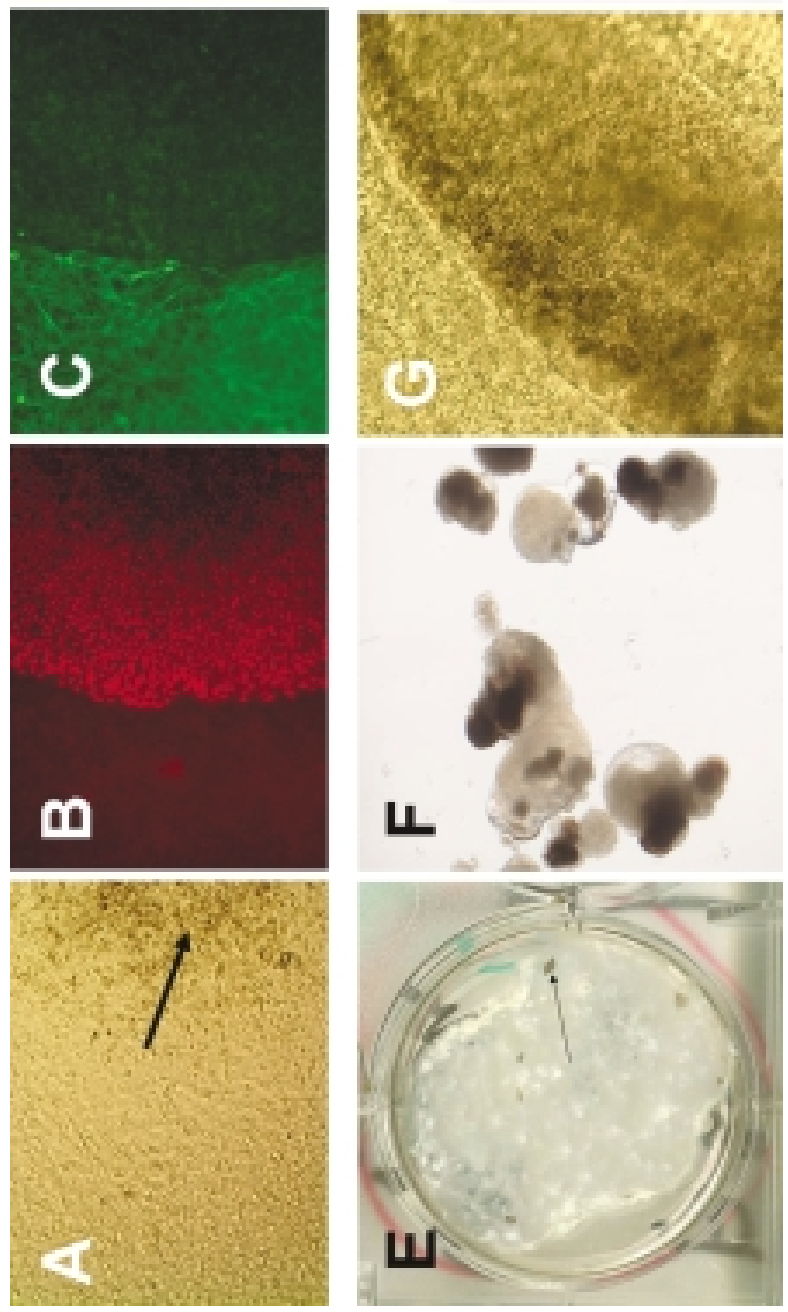

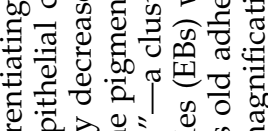

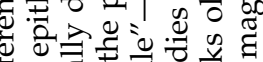

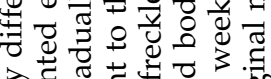

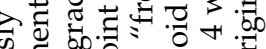

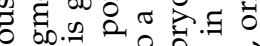

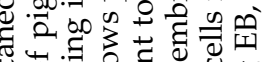
万ิ

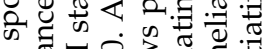

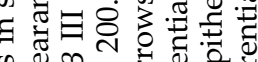

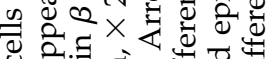

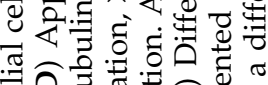

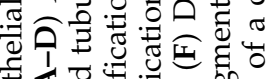

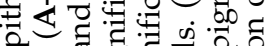
๘

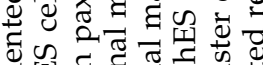
Ф)

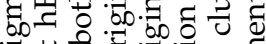

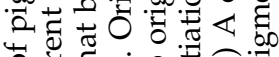

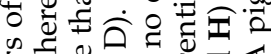

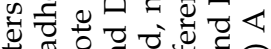

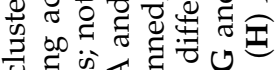
च. \& 0 क क ष

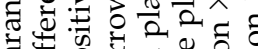
ฮัँ

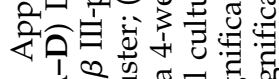

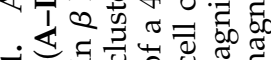
ن.

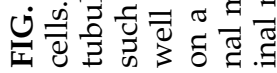



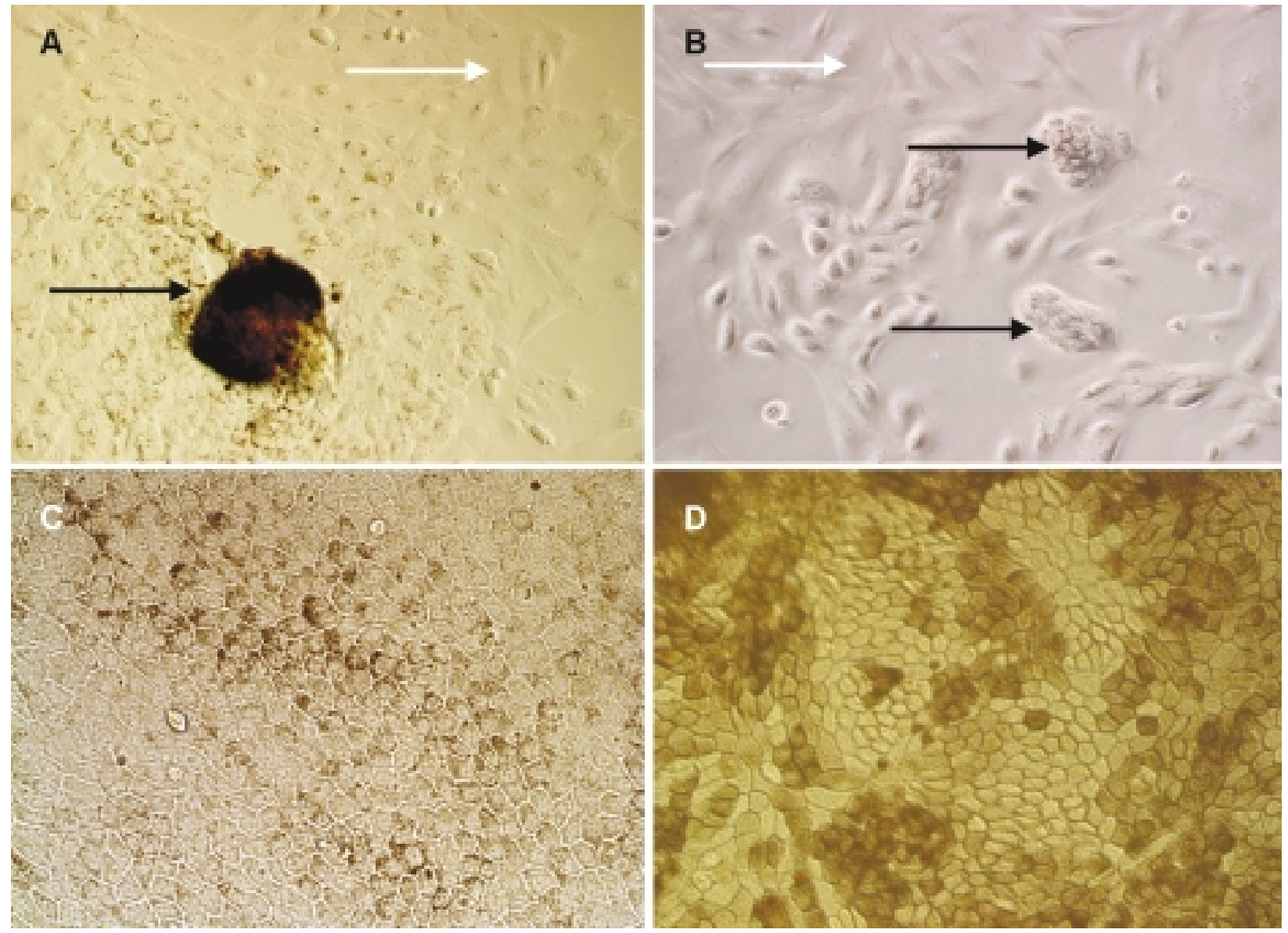

FIG. 2. Loss and restoration of pigmentation and epithelial morphology in culture of hES-derived pigmented cells. (A) Primary embryoid body (EB) outgrowth at 1 week. (B) Primary culture of pigmented epithelial cells, hand-picked from differentiated cultures of human embryonic stem cells at 1 week. (C) Restoration of pigmentation and epithelial morphology in 1-month-old culture. (D) Culture of putative retinal pigment epithalium (RPE) cells after 3 passages. Black arrows in A and B point to the cells still maintaining pigment and epithelial morphology, white arrows show dedifferentiated cells. Note the centrifugal loss of RPE morphology in A. Hoffman modulation optics microscopy, original magnification, $\times 200$.

and analyzing the exclusive intersection between those genes present in hES-RPE/ARPE-19 but not in BE (1026 genes, Fig. 5A). To account for background, we compared this to the exclusive intersection of genes present in BE/hES-RPE, but not ARPE-19 (186 genes, Fig. 5A), which results in a five- to sixfold greater similarity in hES-RPE and ARPE-19 compared to BE. A similar comparison was done for hES-RPE/D407/BE (Fig. 5B), resulting in 760 genes present in hES-RPE and D407 but not in BE versus 196 genes common for hESRPE and BE but not for D407. D407/ARPE-19 appear to lose RPE specific genes, such as RPE65, bestrophin, CRALBP, and PEDF, which is typical of long-term passaged cells (Table 1A). Further data mining revealed known RPE specific on- tologies, such as melanin biosynthesis, vision, and retinol-binding only in fetal RPE and hESRPE but not in ARPE19 (Table 1B).

Comparison of each of hES-RPE, ARPE-19 and D407 to their in vivo counterpart, freshly isolated human fetal RPE (feRPE), was in concordance with other data demonstrating that the transcriptional identity of hES-RPE to human feRPE is significantly greater than that of ARPE-19 (a 1.6-fold difference; 588 genes/364 genes; Fig. 5C) and of D407 (a 2.3-fold difference; 849 genes /373 genes; Fig. 5D). We identified the majority of well-substantiated RPE specific genes present in the hES-RPE data set and absent from ARPE-19 and BE (1186 genes; Fig. 5A) and from D407 and $\mathrm{BE}$ (1452 genes, Fig. 5B), as illustrated further in 

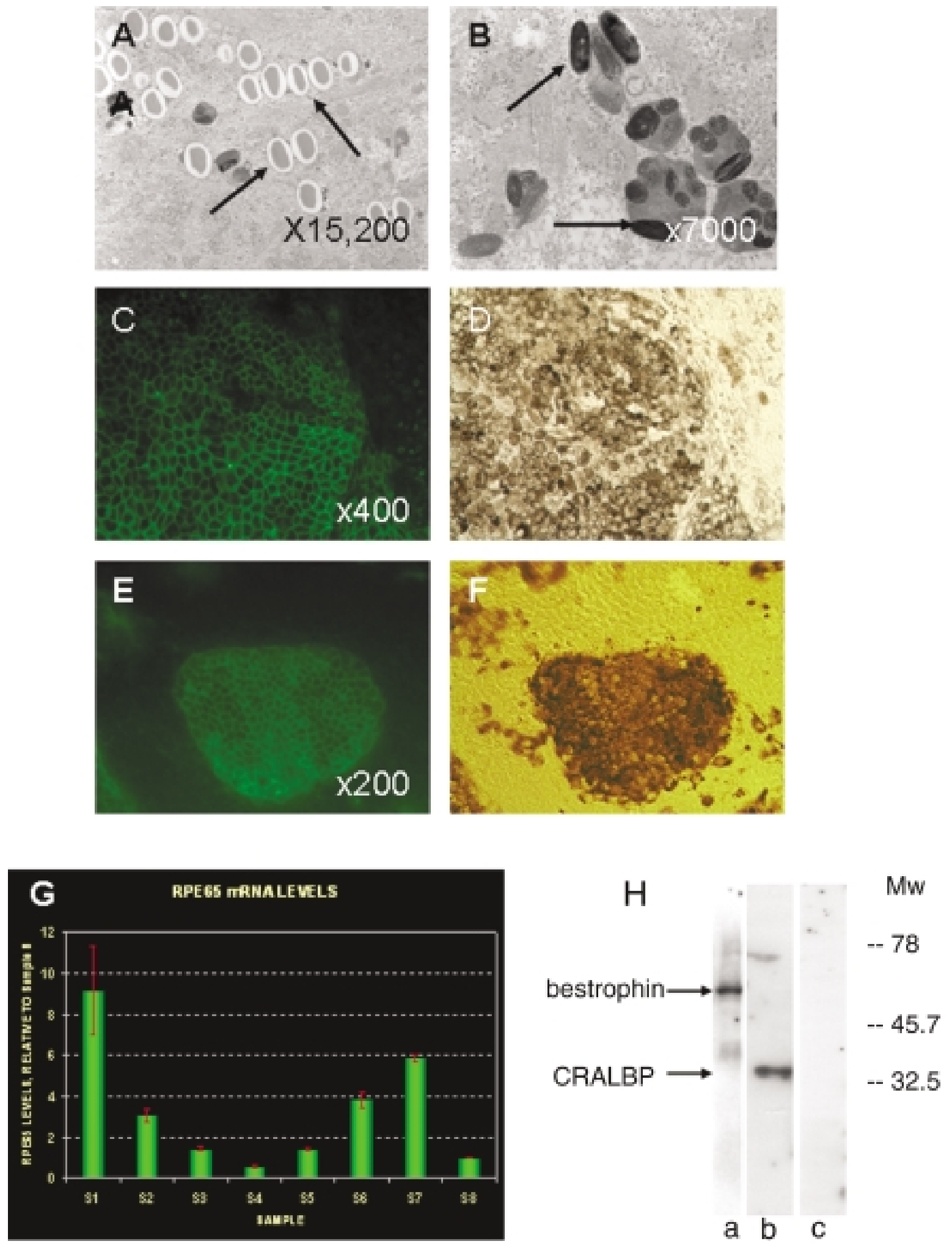

FIG. 3. Assessment of retinal pigment epithelium (RPE) phagocytosis function and molecular markers in human embryonic stem (hES) cell-derived putative RPE original magnification. $(\mathbf{A}) \times 15,200$; $(\mathbf{B}) \times 7000$. (A and B) electron microscopy showing the presence of phagocytozed latex beads inside the RPE-like cell. Arrows show the phagocyotozed latex beads (A) and granules of melanin (B). (C-F) Immunolocalization of RPE markers. (C) bestrophin. (E) Cellular retinaldehyde-binding protein (CRALBP). (D and F) Corresponding phase contrast microscopy fields, original magnification. $(\mathbf{C}$ and $\mathbf{D}) \times 400$. $(\mathbf{E}$ and $\mathbf{F}) \times 200$. Note the localization of both CRALBP and bestrophin to densely pigmented cells. (G) Comparison of RPE65 expression in mature and immature RPE-like cells by real-time RT-PCR. Samples \# 1, 6, and 7 are mature 7-weeks' old cultures; samples \# 2, 3, 4, and 5 are immature 15-days' old cultures; sample \#8 undifferentiated hES cells. (H) Western blot of cell lysates with antibodies to bestrophin (a) and CRALBP (b). (c) Negative control. Molecular weights (mw) 

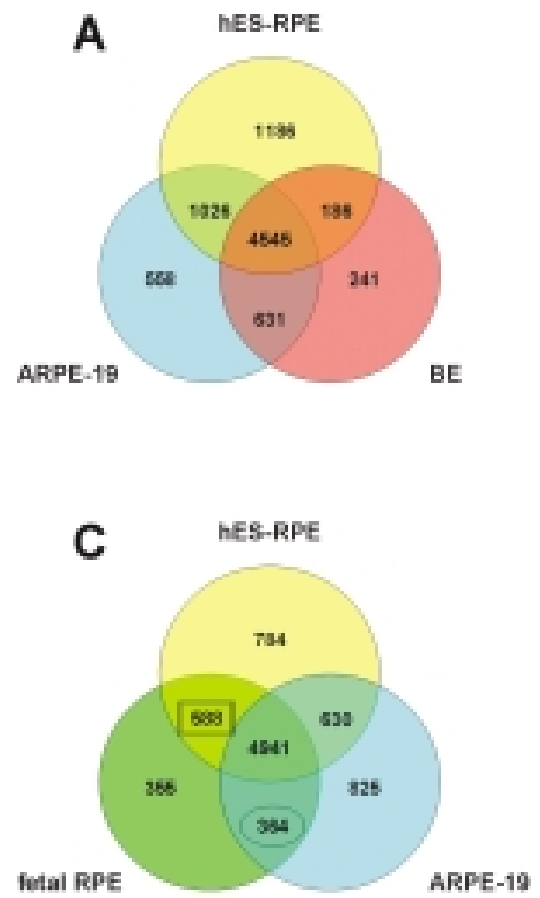
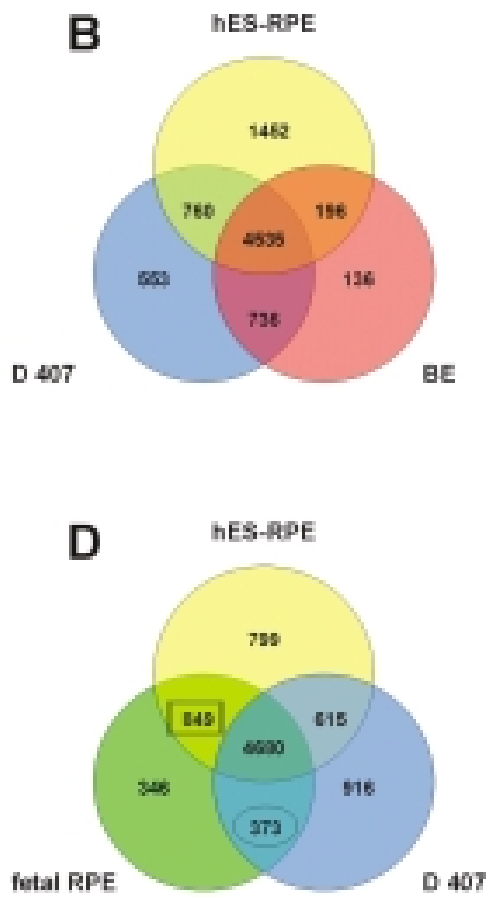

FIG. 4. Venn diagram comparisons of retinal pigment epithelium (RPE) lines. The Venn diagrams demonstrate the transcriptional relationships between human embryonic stem (hES)-RPE and other known RPE cell lines. While ignoring the genes expressed in all three cell types, note the intersections of each Venn diagram because they allow for comparisons of hES-RPE to other RPE cell lines, such as ARPE-19, D407, or feRPE, which serve as positive controls, and bronchial epithelium (BE serves as a negative control. Comparing these intersections to one another, allows one to quantifiably assess the quality of RPE derived from hES. (A) Transcriptional similarity of hES-RPE to ARPE-19 (with 1026 genes in common) and BE (186 genes in common). (B) Although D407 has a similar number of transcripts in common with hES-RPE and BE (760 and 736, respectively) hES-RPE cells (C and D) have a greater transcriptional identity to in vivo-derived RPE relative to ARPE-19 (588, square frame, versus 364, oval frame, genes, see Fig. 4C) and to D407 (849, square frame, versus 373, oval frame, genes, see Fig. 4D).

Table 1A. Such RPE-specific markers identified above, which were only present in hES-RPE and absent in ARPE-19 or D407, were also found in feRPE, demonstrating a higher similarity of hESRPE to its in vivo counterpart than of the cultured RPE lines.

Seven-hundred-and-eighty-four (784) genes present in hES-RPE were absent in the feRPE and ARPE-19 data sets. Since the retention of "stemness" genes could potentially cause transformation of hES derivatives into malignant teratomas if transplanted into patients, we created conservative potential "stemness" genes data using currently available Affymetrix microarray data sets (hES lines H1, H6, H9, and HSF1; Abeyta et al., 2004; Sato et al., 2003). This resulted in a list of 3806 genes present in all 12 data sets (including common housekeeping genes). Only 36 of the 784 genes present in the hES-RPE data set but not the feRPE-ARPE-19 were common to the 3806 potential "stemness" genes. None of these were known "stemness" genes, such as Oct4, Sox2, TDGF1, etc. (Table 2).

\section{Transdifferentiation of $h E S-R P E$}

The ability of RPE to transdifferentiate into retinal neurons and neural progenitors and express the markers of neural lineage, such as pax6 and tubulin $\beta$ III, has been previously described (Fisher and Reh 2001; Reh et al., 1987; Sakaguchi et al., 1997; Vinores et al., 1995; Zhao et al., 1995; Zhao et al., 1997). Similarly, hES-RPE expressed pax6 and tubulin $\beta$ III (Figure 5 A-D) under conditions favoring their proliferation and transdifferentiation. However, once the pigmented epithelial monolayer was reestablished (3-4 weeks after passaging) only a small number of the nonpigmented cells surrounding the pigmented islands remained positive by tubulin $\beta$ III and pax 6 (Fig. 5, E-H). Comparison of transdifferentiated hES-RPE (hES-RPE-TD) to neural precursor mi- 

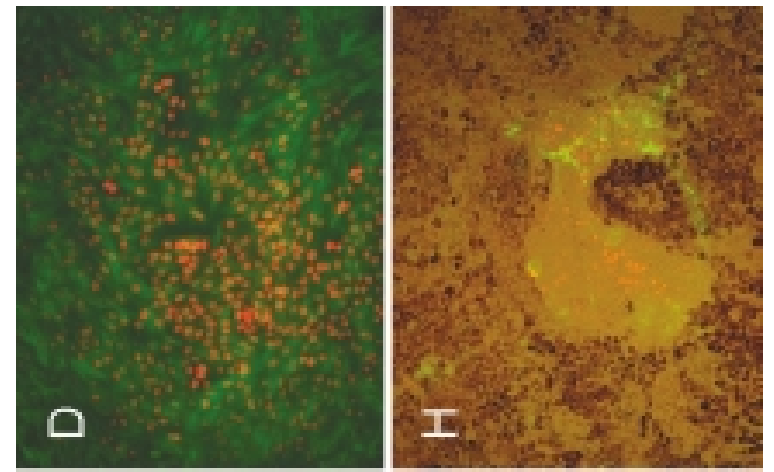

¿ี ฮี

ฮี

कृ

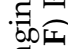

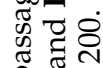

ص $x$

車目. 오

$\sum 0 \pi$

卷:

을
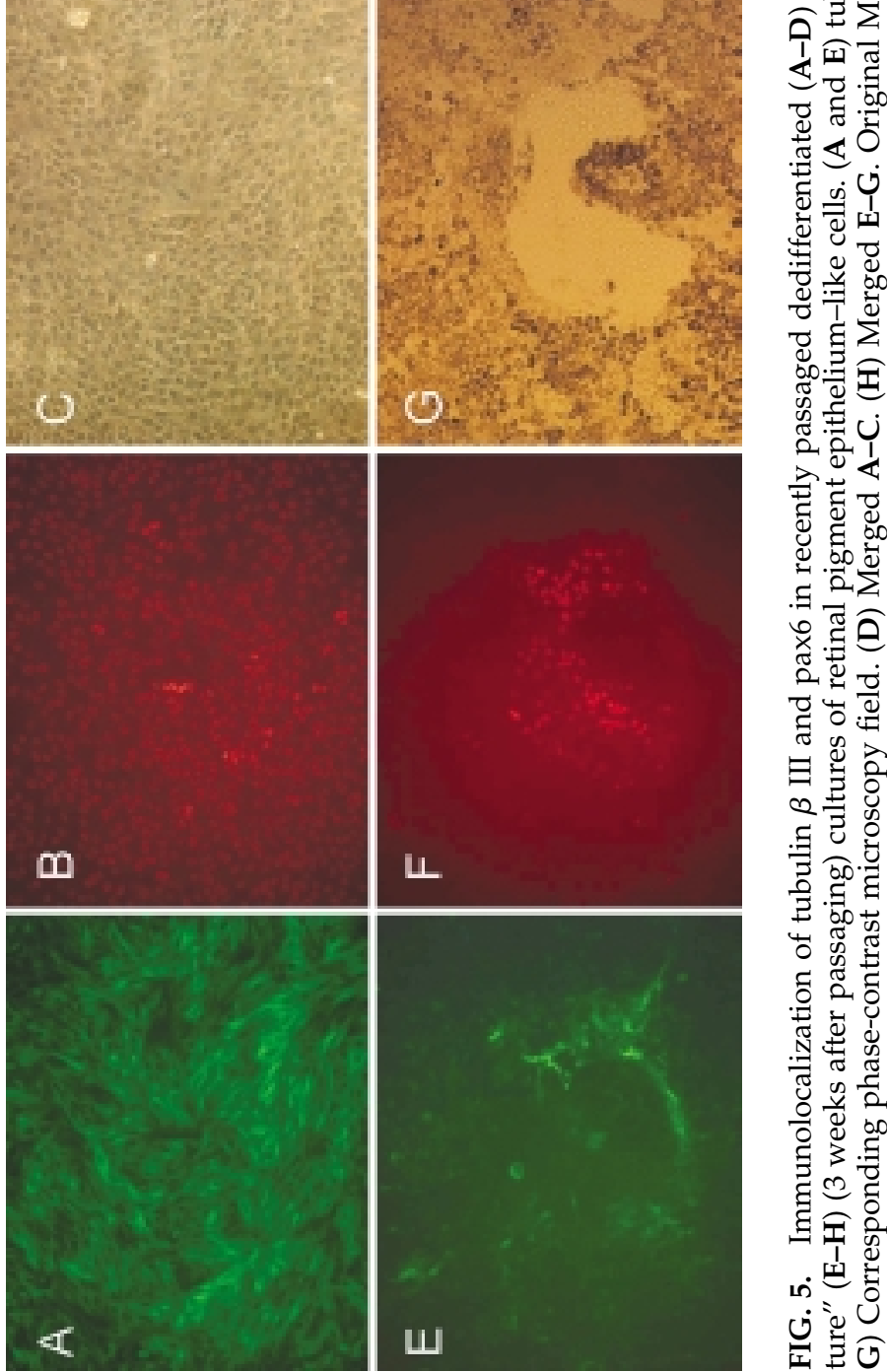


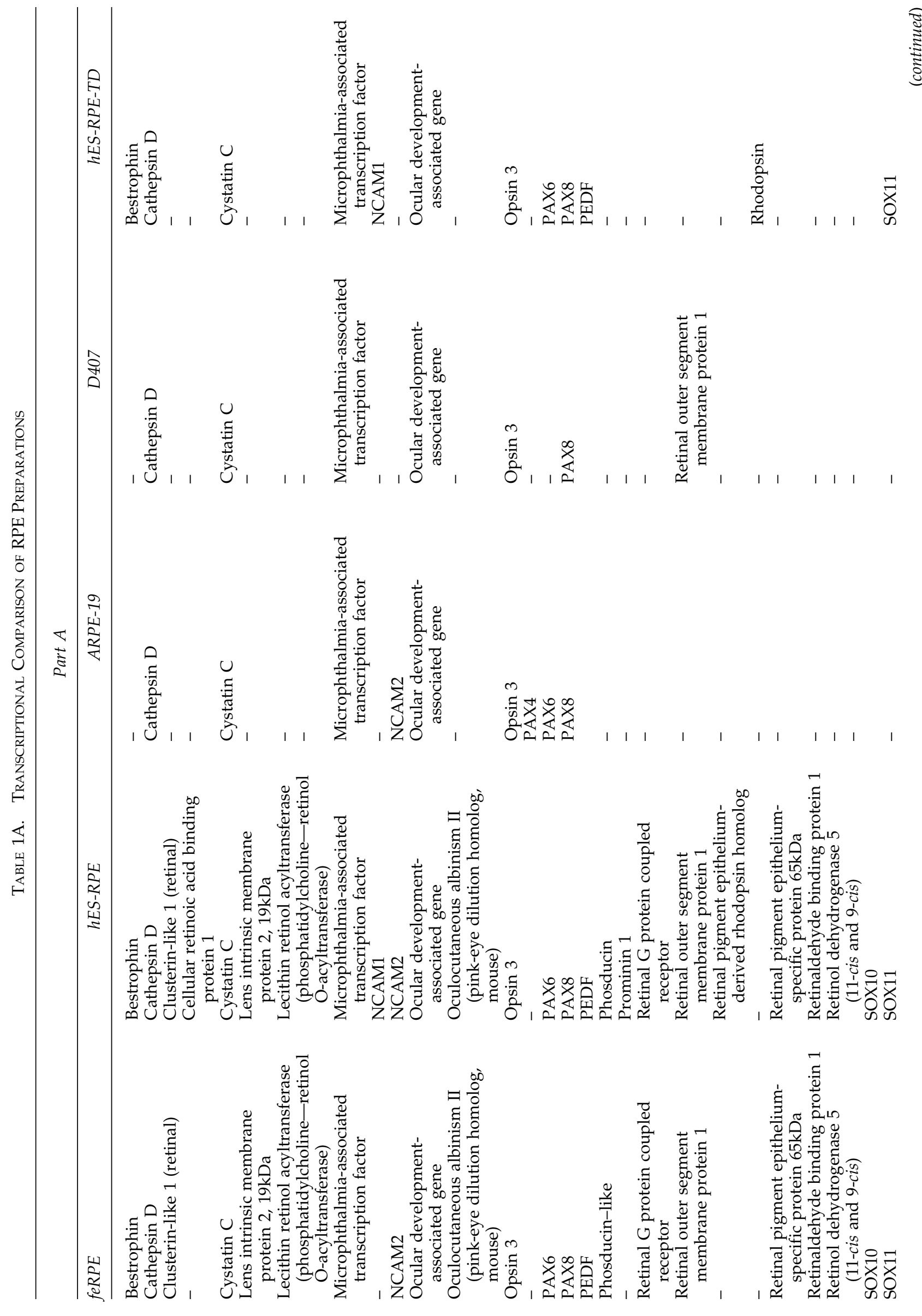




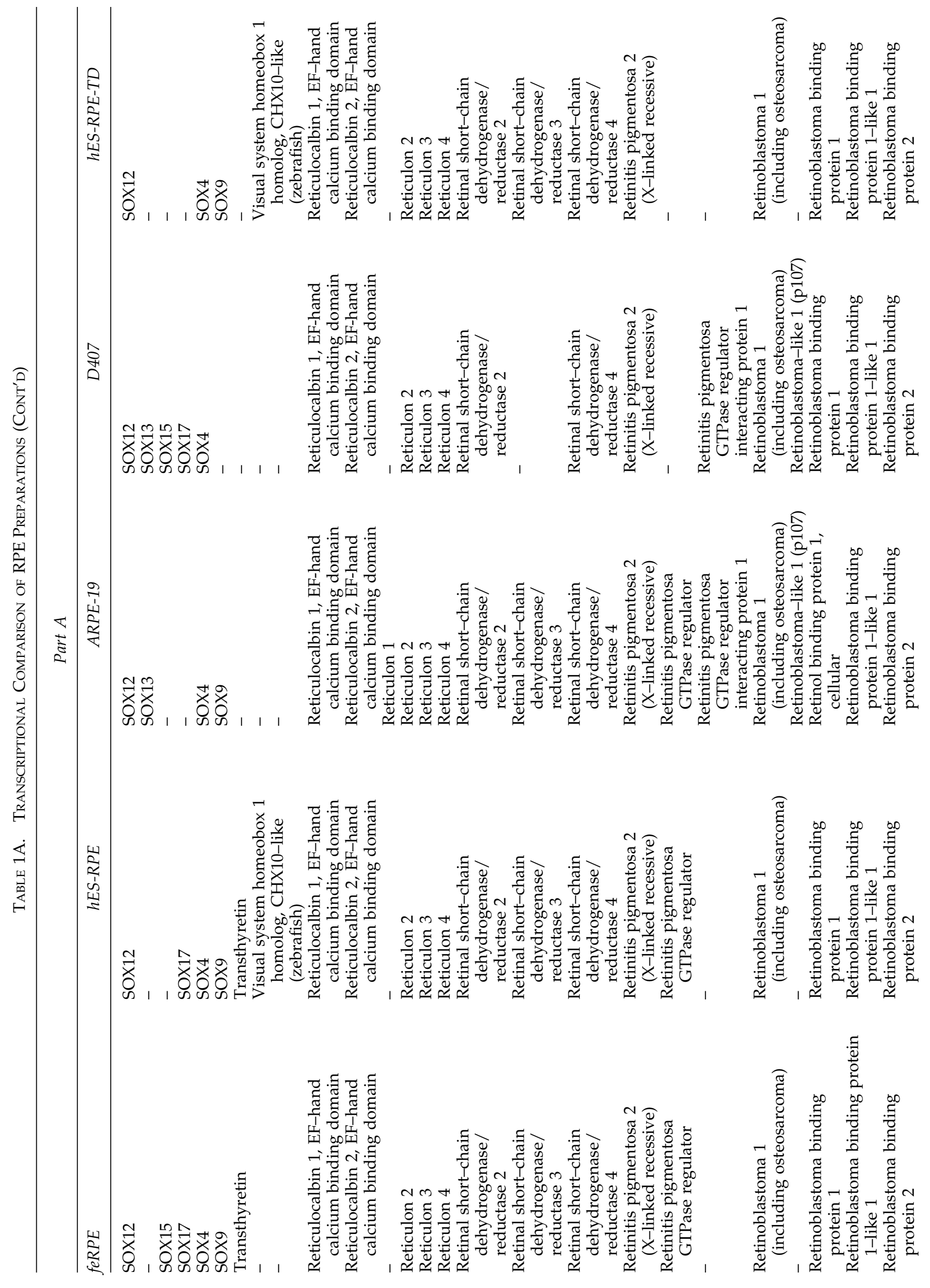




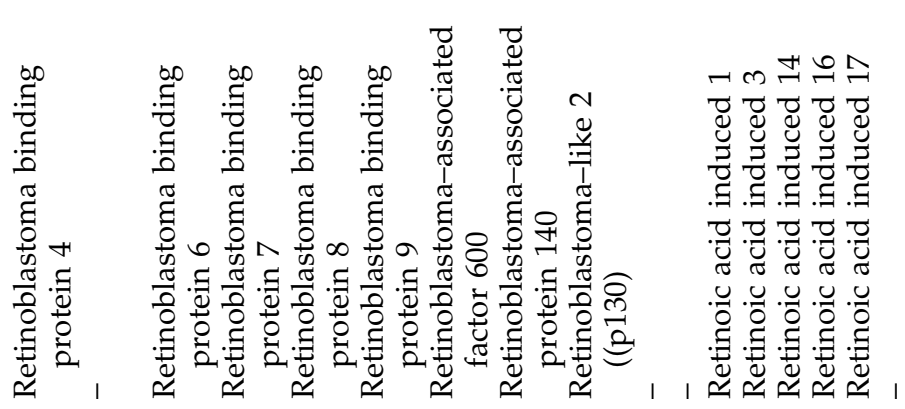

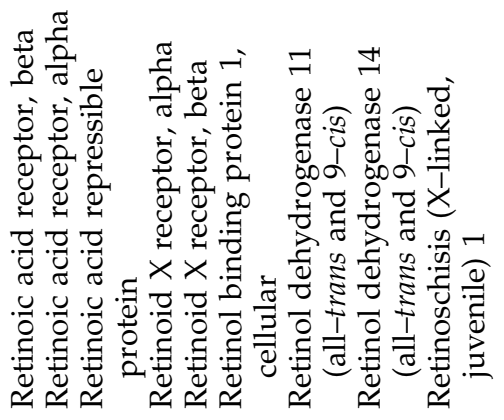

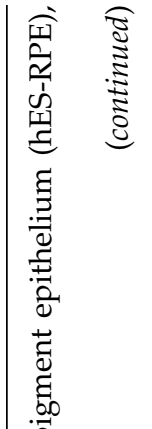

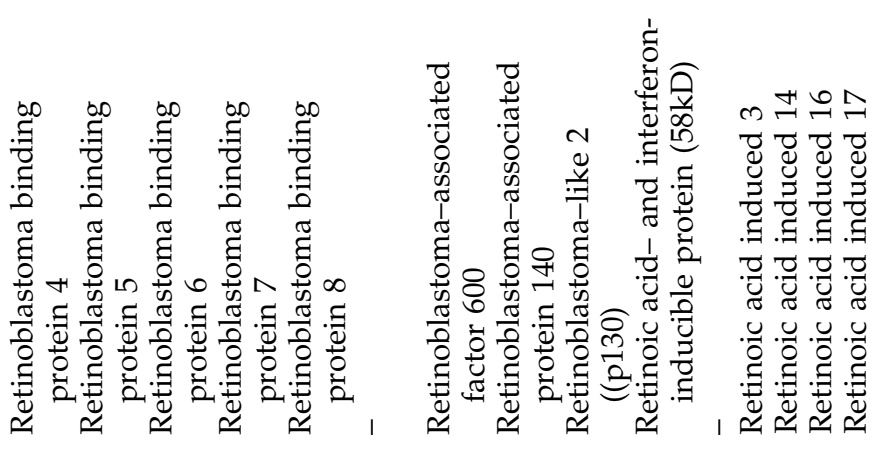
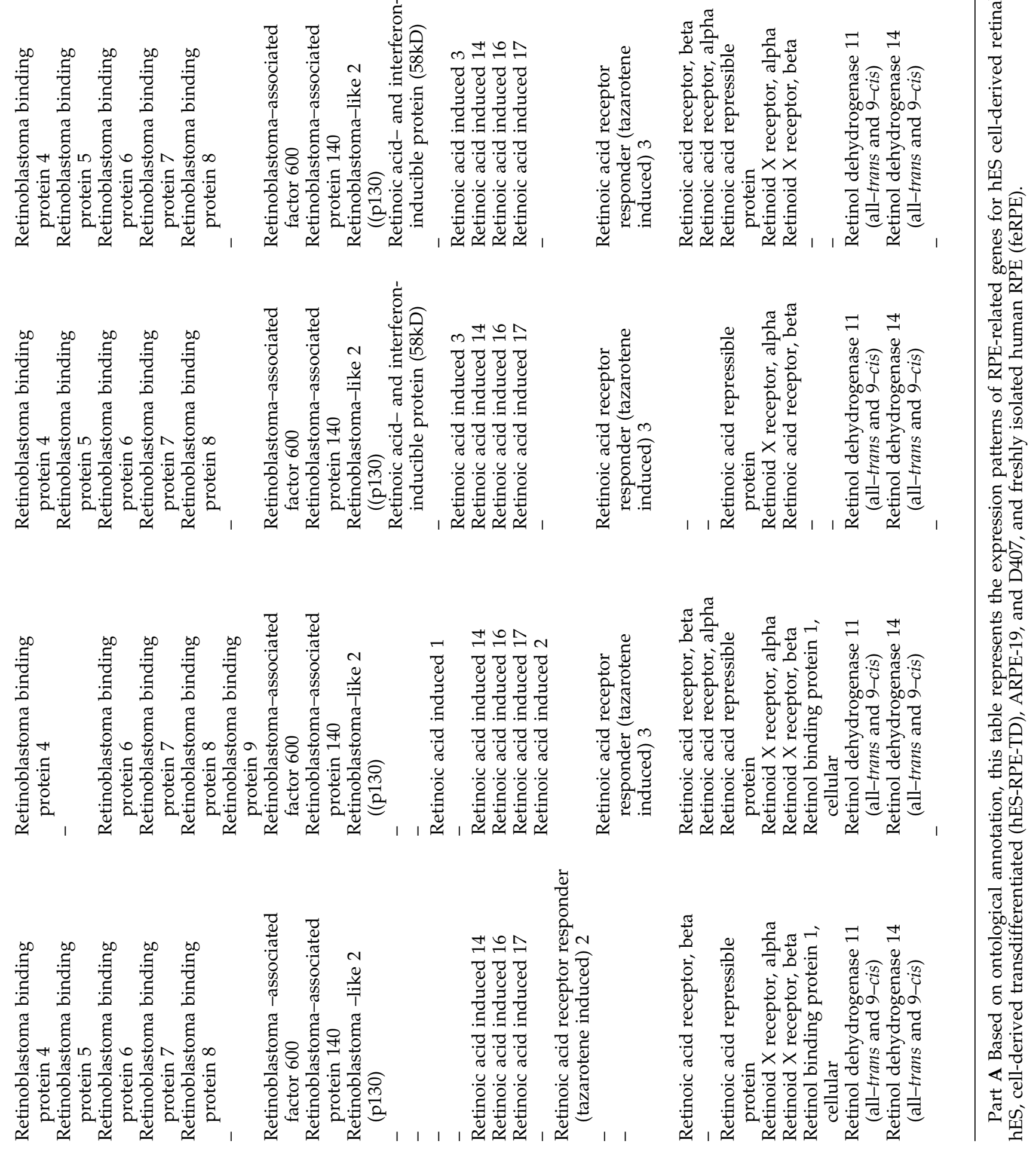


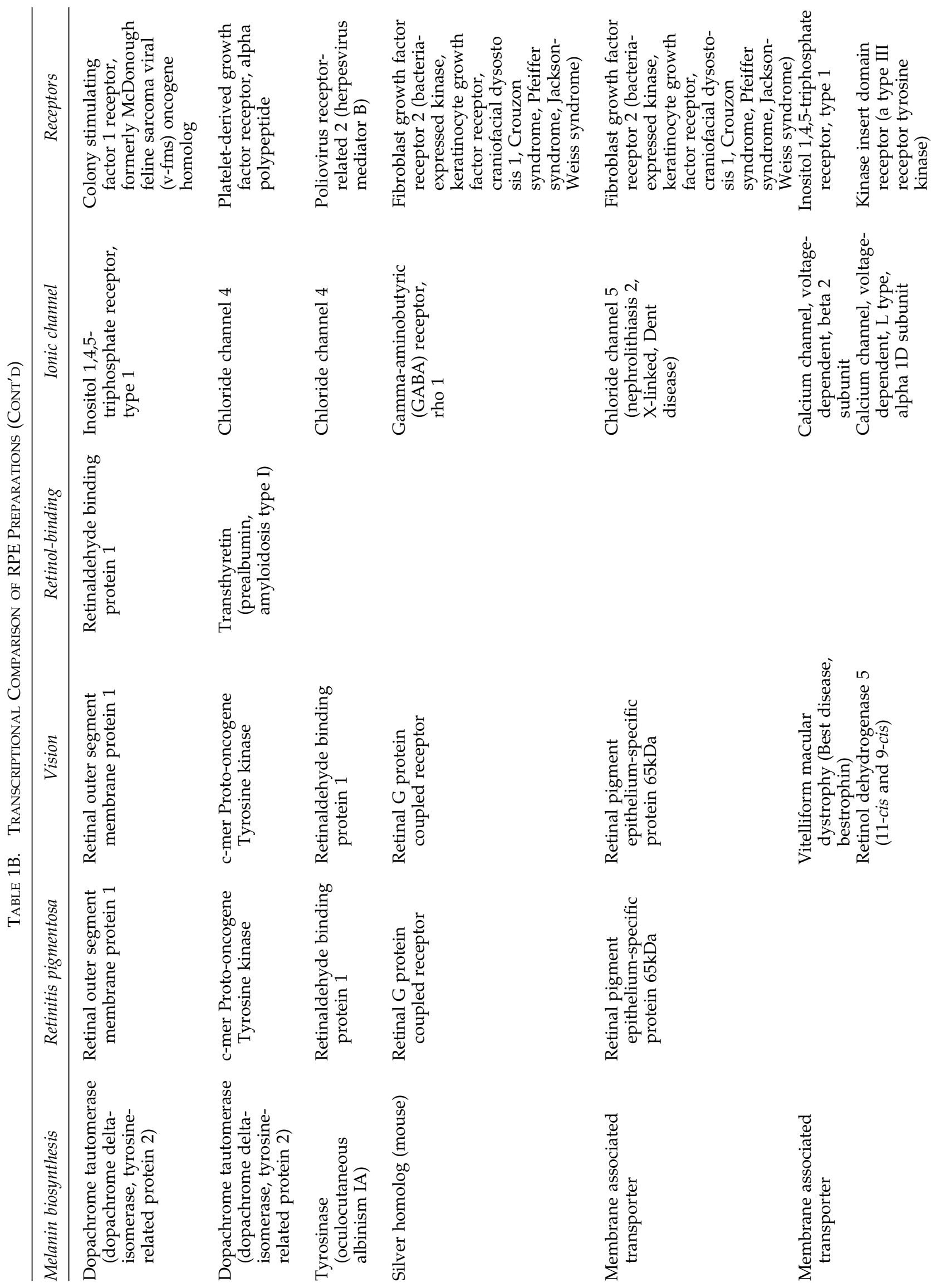



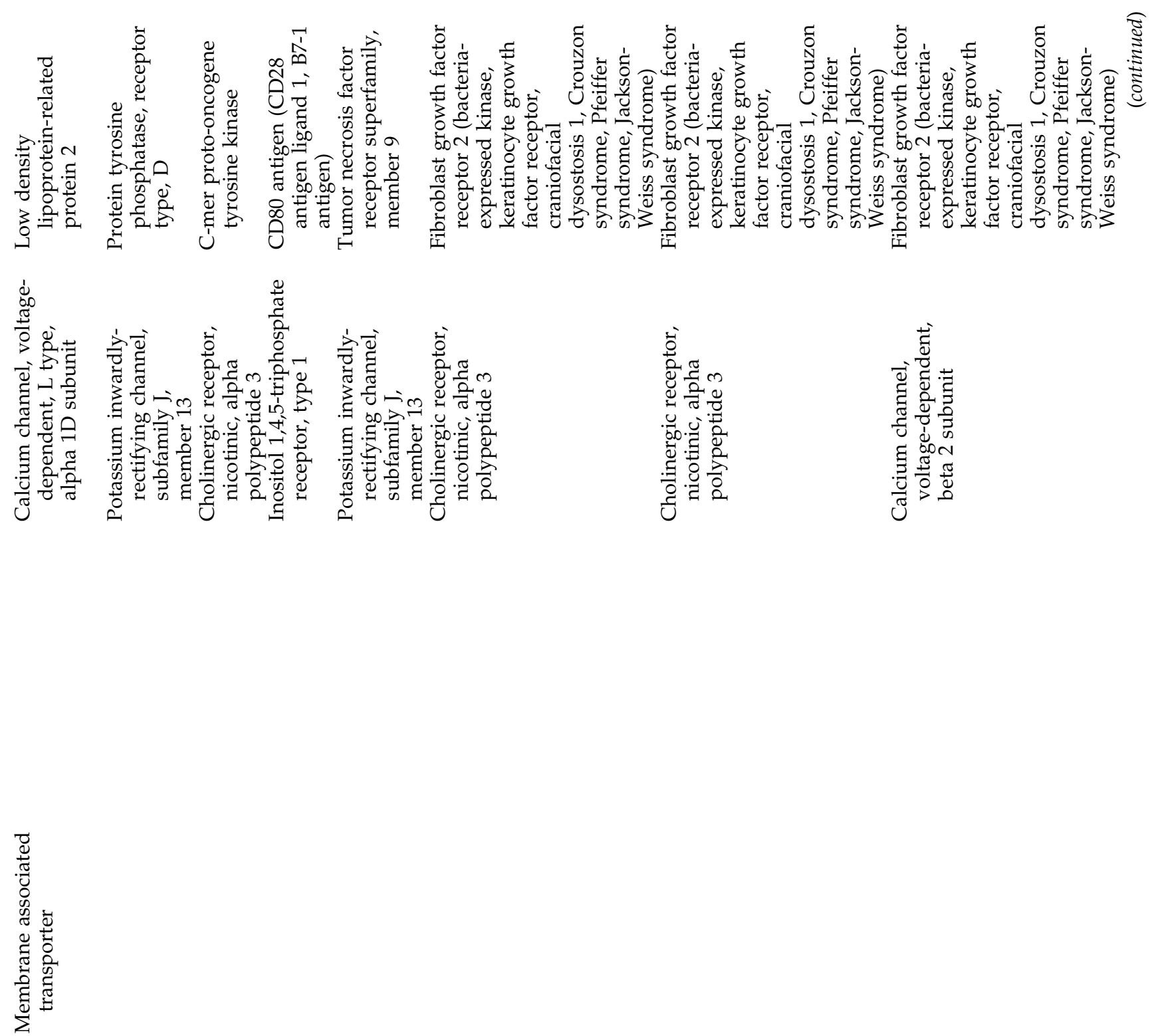


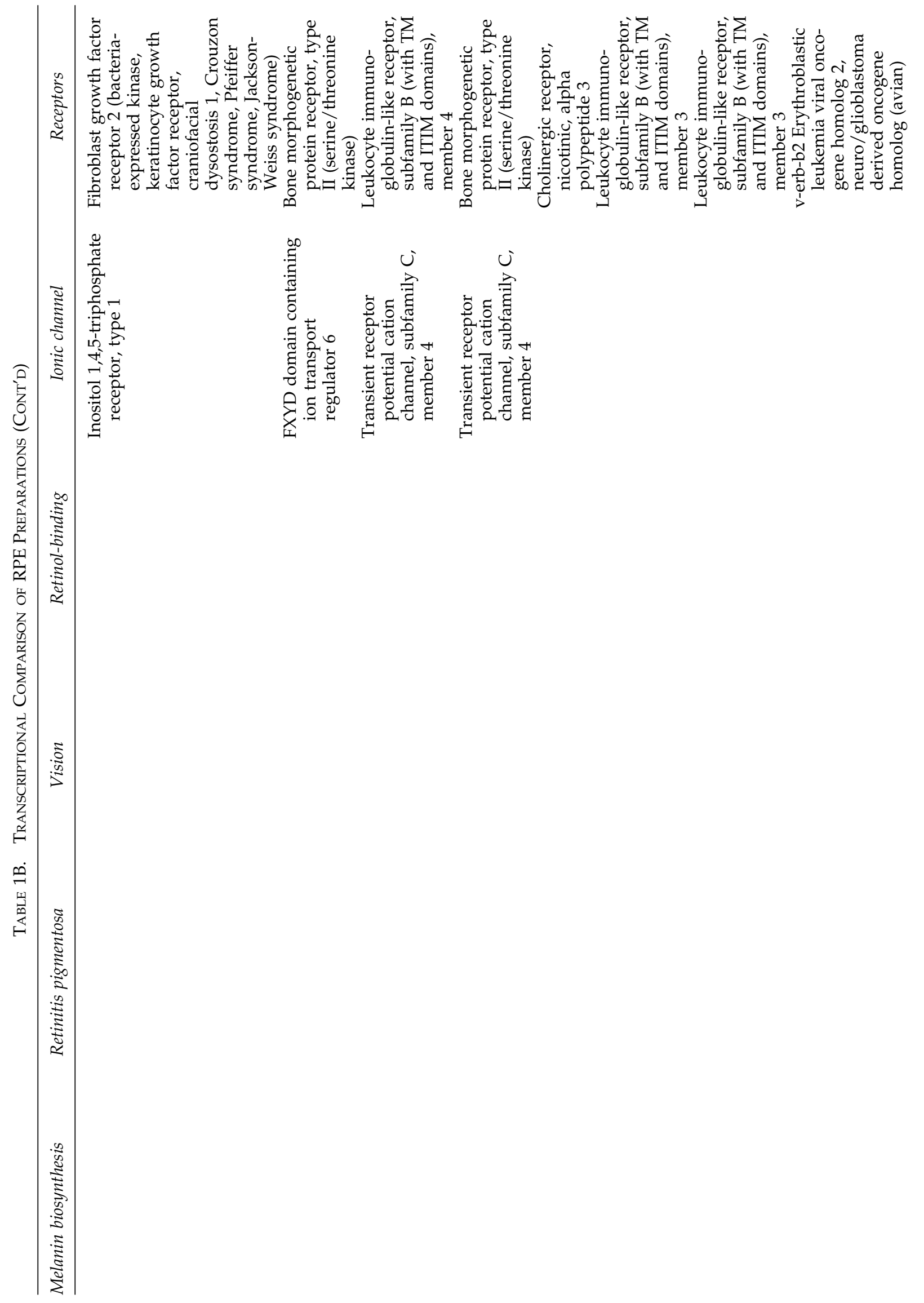




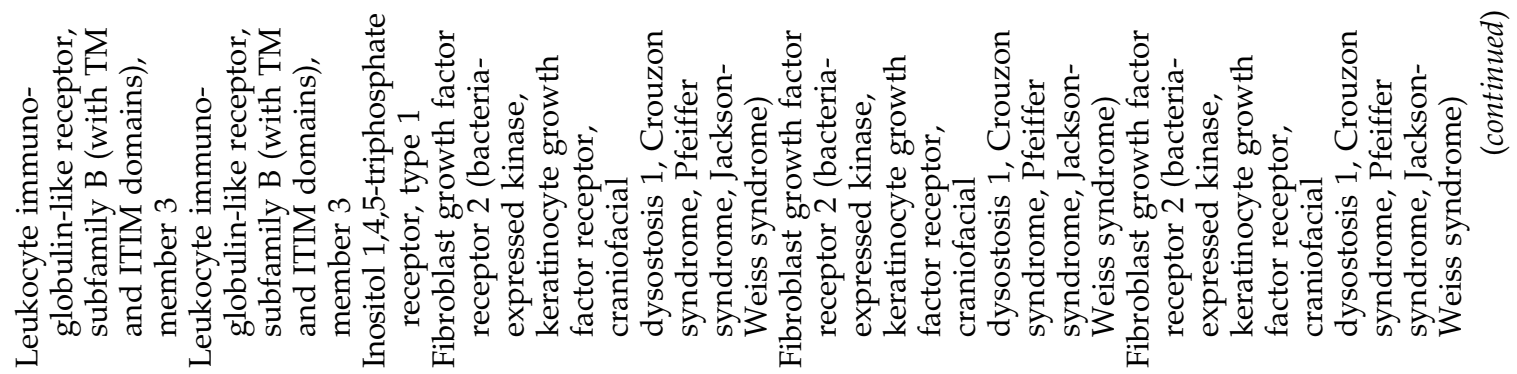




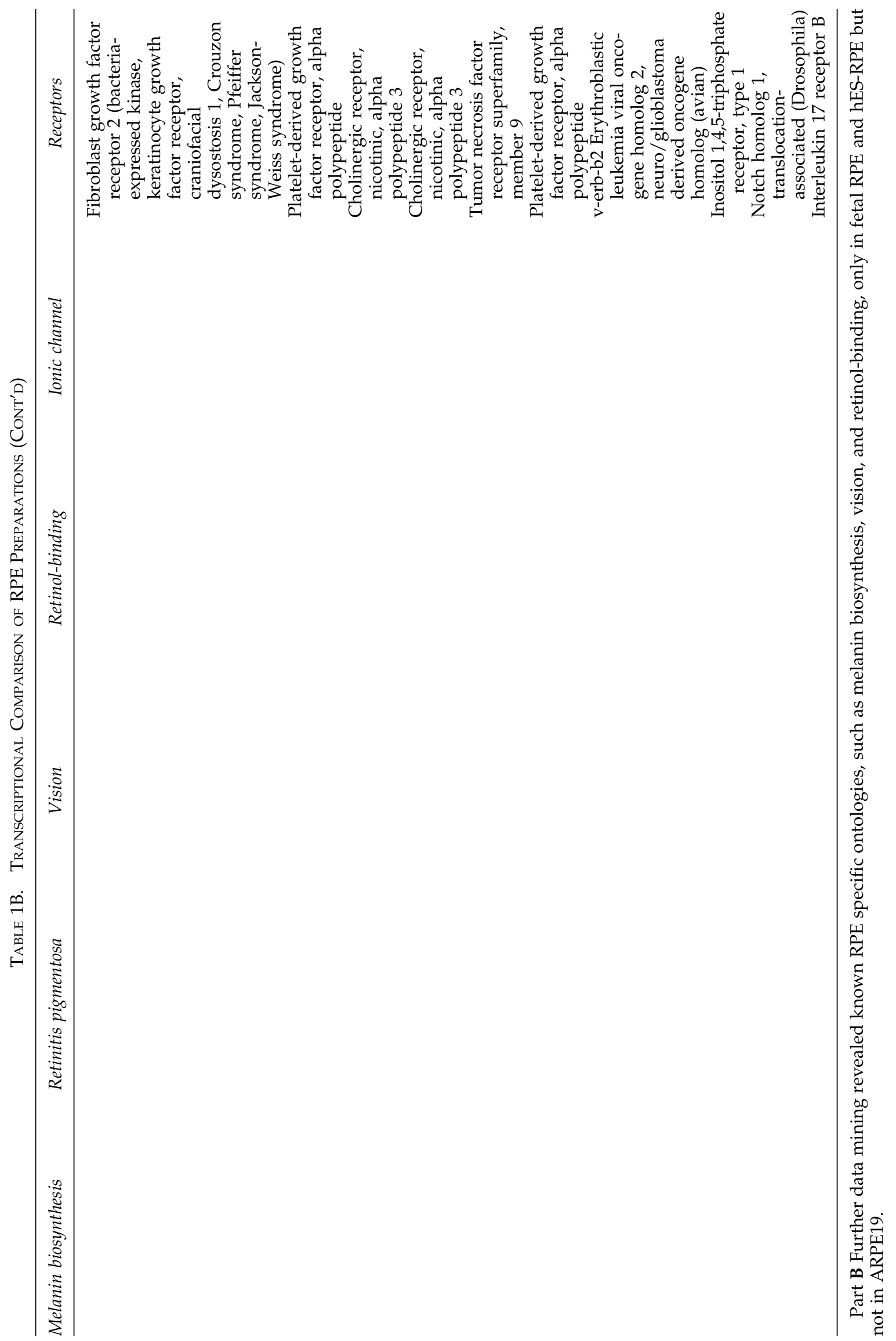


Table 2. Evaluation of hES-RPE for "Stemness" Genes

AQP3
CAB2
CCT6B
CHN1
COL1A2
CRABP1
DCAMKL1
DMBT1
DUSP9
EIF2B3
FBLN1
FKBP1B
FLJ10110
FLJ10156
FLJ11535
FLJ20772
GTF2H4
HLA-DPB1
HSPC109
KIF3C
LLGL2
MGC14258
MMP2
PBX2
PROM1
RBM10
SDF2L1
SELENBP1
SIGIRR
SMARCD2
SPP1
SZF1
TACSTD1
TM4SF11
TXNIP
VAMP8

NOTE: We created a conservative potential "stemness" genes data using currently available Affymetrix microarray data sets (hES lines H1, H6, H9, and HSF1). This resulted in a list of 3806 potential "stemness" genes present in all 12 data sets (including common housekeeping genes). Because the retention of "stemness" genes could potentially cause transformation of hES derivatives into malignant teratomas if transplanted into patients, we took the 784 genes present in hES-RPE and were absent in the feRPE and ARPE-19 data sets which identified only 36 genes in common, none of which were known "stemness" genes such as Oct4, Sox2, TDGF1.

croarray data and to other data sets demonstrated the similarity of hES-RPE-TD to human neural SCs (hNSC; Wright et al. 2003). After filtering out the genes present in hES-RPE, 437 genes were found when hES-RPE-TD data set was linked to neural SCs, including leukemia inhibitory factor receptor, neural cell adhesion molecule 1 , and neurotrophic tyrosine kinase, receptor, type 2 and type 3, and most of RPE-specific genes were downregulated (Table 3).

\section{DISCUSSION}

Neurosensory retina and retinal pigment epithelium share the same bipotential neuroepithelial progenitor in the growing optic vesicle. Their determination requires the activities of pax2, pax6, and mitf (Baumer et al., 2003). At earlier stages, pax6 acts as an activator of proneural genes and is downregulated in the RPE in further development, remaining in amacrine and ganglion cells in mature retina (reviewed by AsheryPadan and Gruss, 2001). Previous studies have demonstrated that ES cells can be differentiated in culture into neurons and neuroectodermal progenitors (Carpenter et al., 2001; Kawasaki et al., 2002; Zhao et al., 2002), including retinal neurons that can differentiate into photoreceptor-like structures (Zhao et al., 2002). In our experiments, cells of neural lineage were detected in differentiating cultures of ES cells as evidenced by immunostaining with anitibodies to pax6, tubulin $\beta$ III, nestin (not shown). Interestingly, many pax6 and tubulin $\beta$ III-positive cells were surrounding forming pigmented epithelial clusters, and their expression gradually disappeared towards the more densely pigmented centers, suggesting the presence of transitory phenotypes (Fig. 1, A-D). Our data suggest that differentiation of hES cells into RPE is a further progression of initial neuronal lineage stage.

Ying and coauthors (2003) have shown that commitment to the neuronal lineage of murine ES cells depends upon autocrine FGF (Fibroblast growth factor). The present study suggests that the differentiation of hES cells in the absence of exogenous factors proceeds beyond their commitment to the neuroectodermal lineage, resulting in the appearance of putative retinal pigment epithelial cells. Previous reports of the appearance of pigmented epithelial cells in cultures of differentiating primate ES cells (Haruta et al., 2004; Hirano et al., 2003; Kawasaki et al., 2002) suggested that such differentiation to neurons and ocular tissues was attributed to stromal cellderived inducing activity (SDIA) coming from cocultured mouse PA6 cells. However, we have obtained consistent differentiation of human ES cells to RPE-like cells to be independent of animal coculture, including long-term hES cultures grown either on feeder layers or feeder-free on gelatin, fibronectin, laminin, collagen types I and IV, or in EBs. Moreover, hES cells passaged without feeder cells produced pigmented epithelial 
Table 3. Transcripts Common to Human Neural Stem Cells and hES-RPE-TD

A kinase (PRKA) anchor protein (gravin) 12

Adaptor-related protein complex 1, beta 1 subunit

Adaptor-related protein complex 1 , sigma 1 subunit

Adaptor-related protein complex 3, mu 2 subunit

Adenomatous polyposis coli like

Adenylate kinase 1

ADP-ribosylation factor 5

ADP-ribosylation factor GTPase activating protein 1

ADP-ribosylation factor-like 7

ADP-ribosylation-like factor 6 interacting protein 4

Amino-terminal enhancer of split

Angio-associated, migratory cell protein

Angiopoietin 1

Angiopoietin-like 4

Antigen identified by monoclonal antibody Ki-67

Apoptosis related protein APR-3

ARF-GAP, RHO-GAP, ankyrin repeat and plekstrin homology domains-containing protein 3

ArsA arsenite transporter, ATP-binding, homolog 1 (bacterial)

ASF1 anti-silencing function 1 homolog B (Saccharomyces cerevisiae)

ATP-binding cassette, sub-family F (GCN20), member 2

Aurora kinase B

Autophagin-1

B-cell RAG associated protein

Baculoviral IAP repeat-containing 5 (survivin)

B-cell CLL/lymphoma 9

BCL2-antagonist of cell death

BCL2-associated $X$ protein

Branched chain alpha-ketoacid dehydrogenase kinase

Bridging integrator 3

BUB1 budding uninhibited by benzimidazoles 1 homolog (yeast)

BUB1 budding uninhibited by benzimidazoles 1 homolog beta (yeast)

Cadherin 6, type 2, K-cadherin (fetal kidney)

Calmodulin binding transcription activator 2

Calpain 5

Calsequestrin 1 (fast-twitch, skeletal muscle)

cAMP responsive element binding protein 5

Carbohydrate (N-acetylglucosamine-6-O) sulfotransferase 2

Carnitine palmitoyltransferase 1A (liver)

Cation-transporting ATPase

CCAAT/enhancer binding protein $(\mathrm{C} / \mathrm{EBP})$, gamma

CD151 antigen

CDC42 binding protein kinase alpha (DMPK-like)

CDC-like kinase 3

Cell division cycle 2-like 1 (PITSLRE proteins)

Cell division cycle 34

Centaurin, delta 1

Centromere protein $\mathrm{A}, 17 \mathrm{kDa}$

Centromere protein $\mathrm{B}, 80 \mathrm{kDa}$

Centromere protein F, 350/400ka (mitosin)

Chondroitin polymerizing factor

Chromosome 11 hypothetical protein ORF4

Chromosome 14 open reading frame 104

Chromosome 14 open reading frame 133

Chromosome 14 open reading frame 94

Chromosome 16 open reading frame 7

Chromosome 18 open reading frame 1

Chromosome 20 open reading frame 14

Chromosome 20 open reading frame 27

Chromosome 21 open reading frame 45

Chromosome 6 open reading frame 130

Chromosome 6 open reading frame 139

Chromosome 6 open reading frame 18

Chromosome condensation protein $\mathrm{G}$

Chromosome $\mathrm{X}$ open reading frame 9 
Table 3. Transcripts Common to Human Neural Stem Cells and hes-RPE-TD (Cont'd)

CK2 interacting protein 1; HQ0024c protein

Clathrin, light polypeptide (Lcb)

Cleavage and polyadenylation specific factor $4,30 \mathrm{kDa}$

Cleft lip and palate associated transmembrane protein 1

Coatomer protein complex, subunit epsilon

Cockayne syndrome 1 (classical)

Collapsin response mediator protein 1

Component of oligomeric golgi complex 4

COP9 constitutive photomorphogenic homolog subunit 7B (Arabidopsis)

CP110 protein

Cyclin B1

Cyclin B2

Cyclin D3

Cyclin E2

Cyclin-dependent kinase 5

Cyclin-dependent kinase 5, regulatory subunit 1 (p35)

Cyclin-dependent kinase inhibitor 1A (p21, Cip1)

Cytochrome c-1

Cytoplasmic linker 2

D4, zinc and double PHD fingers family 2

Damage-specific DNA binding protein 2, 48kDa

DC12 protein

DEAD (Asp-Glu-Ala-Asp) box polypeptide 46

Death-associated protein kinase 1

Deformed epidermal autoregulatory factor 1 (Drosophila)

Deleted in lymphocytic leukemia, 1

Dihydropyrimidinase-like 3

Discs, large homolog 7 (Drosophila)

DKFZP586J0619 protein

DNA (cytosine-5-)-methyltransferase 2

DNA glycosylase hFPG2

DNA replication factor

DNA segment on chromosome 10 (unique) 170

DNA2 DNA replication helicase 2-like (yeast)

Dolichyl-phosphate mannosyltransferase polypeptide 2, regulatory subunit

Dual specificity phosphatase 6

Dual-specificity tyrosine-(Y)-phosphorylation regulated kinase 2

Dudulin 2

Dynactin 2 (p50)

E2F transcription factor 1

E2F transcription factor 3

E2F transcription factor 5, p130-binding

E2IG2 protein

Ectodermal-neural cortex (with BTB-like domain)

Egl nine homolog 2 (Caenorhabditis elegans)

EH-domain containing 1

EH-domain containing 2

Emerin (Emery-Dreifuss muscular dystrophy)

Enigma (LIM domain protein)

Ephrin-B2

Epithelial cell transforming sequence 2 oncogene

Epithelial membrane protein 3

ets Variant gene 5 (ets-related molecule)

ets Variant gene 7 (TEL2 oncogene)

Ets2 repressor factor

Eukaryotic translation initiation factor $2 \mathrm{~B}$, subunit 4 delta, $67 \mathrm{kDa}$

Exosome complex exonuclease RRP41

Exostoses (multiple)-like 3

Extra spindle poles like 1 (S. cerevisiae)

Family with sequence similarity 16, member A, X-linked

Fanconi anemia, complementation group G

Fasciculation and elongation protein zeta 1 (zygin I)

Fascin homolog 1, actin-bundling protein (Strongylocentrotus purpuratus)

Fatty-acid desaturase 2 
Table 3. Transcripts Common to Human Neural Stem Cells and hes-RPE-TD (Cont’d)

Fatty-acid synthase

Ferredoxin reductase

Fibroblast growth factor 1 (acidic)

Fibrosin 1

Flap structure-specific endonuclease 1

Forkhead box M1

Four jointed box 1 (Drosophila)

Fzr1 protein

G protein pathway suppressor 1

Galactose-1-phosphate uridylyltransferase

Geminin, DNA replication inhibitor

GLI-Kruppel family member GLI2

Glucosidase, alpha; acid (Pompe disease, glycogen storage disease type II)

Glucosidase, beta; acid (includes glucosylceramidase)

Glutamate dehydrogenase 1

Glutaminyl-peptide cyclotransferase (glutaminyl cyclase)

Glycine receptor, alpha 1 (startle disease/hyperekplexia, stiff man syndrome)

Glypican 1

gp25L2 Protein

Guanine nucleotide binding protein (G protein), alpha inhibiting activity polypeptide 2

Guanine nucleotide binding protein (G protein), beta 5

Guanine nucleotide binding protein ( $\mathrm{G}$ protein), beta polypeptide 2

Guanylate kinase 1

H1 histone family, member $X$

Heat shock protein 75

Heparan sulfate (glucosamine) 3-O-sulfotransferase 3A1

Hepatitis delta antigen-interacting protein A

Heterogeneous nuclear ribonucleoprotein A3

High-mobility group AT-hook 1

High-mobility group 20B

Histamine receptor $\mathrm{H} 1$

Histone 1, H2bd

Histone 1, H4c

HIV-1 Tat interactive protein, $60 \mathrm{kDa}$

HLA-B associated transcript 2

HLA-B associated transcript 8

HMT1 hnRNP methyltransferase-like 2 (Saccharomyces cerevisiae)

Host-cell factor $\mathrm{C} 1$ regulator 1 (XPO1 dependant)

HSPC023 protein

Hyaluronan-mediated motility receptor (RHAMM)

Hypothetical protein AF053356_CDS3

Hypothetical protein BC002926

Hypothetical protein DKFZp434H1419

Hypothetical protein FLJ10120

Hypothetical protein FLJ10439

Hypothetical protein FLJ10597

Hypothetical protein FLJ10719

Hypothetical protein FLJ11773

Hypothetical protein FLJ11795

Hypothetical protein FLJ12443

Hypothetical protein FLJ12750

Hypothetical protein FLJ12788

Hypothetical protein FLJ12886

Hypothetical protein FLJ13511

Hypothetical protein FLJ14084

Hypothetical protein FLJ14153

Hypothetical protein FLJ20340

Hypothetical protein FLJ20485

Hypothetical protein FLJ20546

Hypothetical protein FLJ20551

Hypothetical protein FLJ20647

Hypothetical protein FLJ21127

Hypothetical protein FLJ21172

Hypothetical protein FLJ21816 
Table 3. Transcripts Common to Human Neural Stem Cells and hes-RPE-TD (Cont'd)

Hypothetical protein FLJ22054

Hypothetical protein FLJ22169

Hypothetical protein FLJ22202

Hypothetical protein FLJ22329

Hypothetical protein FLJ22965

Hypothetical protein FLJ23436

Hypothetical protein FLJ23548

Hypothetical protein FLJ38993

Hypothetical protein MGC2656

Hypothetical protein MGC3047

Hypothetical protein MGC4172

Hypothetical protein MGC4293

Hypothetical protein MGC4368

Inhibitor of DNA binding 2, dominant negative helix-loop-helix protein

Inhibitor of growth family, member 1

Inositol polyphosphate phosphatase-like 1

Insulin-like growth factor binding protein $2,36 \mathrm{kDa}$

Insulinoma-associated 1

Integrin, alpha 10

Integrin-linked kinase

Interferon, alpha-inducible protein (clone IFI-15K)

KIAA0056 protein

KIAA0090 protein

KIAA0095 gene product

KIAA0100 gene product

KIAA0101 gene product

KIAA0117 protein

KIAA0186 gene product

KIAA0195 gene product

KIAA0196 gene product

KIAA0218 gene product

KIAA0323 protein

KIAA0537 gene product

KIAA0664 protein

KIAA0773 gene product

KIAA1068 protein

KIAA1115 protein

Kinesin family member 11

Kinesin family member 14

Kinesin family member 23

Kinesin family member $4 \mathrm{~A}$

Kinesin-like 7

KIT ligand

Lamin B1

Lectin, galactoside-binding, soluble, 3 (galectin 3)

Leucine-rich repeat containing 17

Leucine zipper domain protein

LGN protein

Likely ortholog of mouse embryonic epithelial gene 1

Linked to Surfeit genes in Fugu rubripes 2

Mannosidase, alpha, class 1B, member 1

Maternal embryonic leucine zipper kinase

Matrix metalloproteinase 16 (membrane-inserted)

MCM5 minichromosome maintenance-deficient 5, cell division cycle 46 (Saccharomyces cerevisiae)

MCM7 minichromosome maintenance-deficient 7 (Saccharomyces cerevisiae)

Mediator of RNA polymerase II transcription, subunit 6 homolog (yeast)

Metallothionein $1 \mathrm{H}$

Methyl-CpG binding domain protein 3

Methylene tetrahydrofolate dehydrogenase (NAD+ dependent), methenyltetrahydrofolate cyclohydrolase

Methyltransferase-like 1

Microspherule protein 1

Mitochondrial ribosomal protein L12

Mitochondrial ribosomal protein L46

Mitochondrial ribosomal protein S12 
Table 3. Transcripts Common to Human Neural Stem Cells and hes-RPE-TD (Cont'd)

Mitogen-activated protein kinase associated protein 1

Mitogen-activated protein kinase kinase 2

Mitogen-activated protein kinase kinase 3

M-phase phosphoprotein 1

Mucosa associated lymphoid tissue lymphoma translocation gene 1

Myeloid/lymphoid or mixed-lineage leukemia (trithorax homolog, Drosophila); translocated to, 3

Myosin IXB

Myosin, heavy polypeptide 9, nonmuscle

N-deacetylase/N-sulfotransferase (heparan glucosaminyl) 1

NEDD9 interacting protein with calponin homology and LIM domains

N-ethylmaleimide-sensitive factor attachment protein, alpha

N-ethylmaleimide-sensitive factor attachment protein, gamma

Neural precursor cell expressed, developmentally downregulated 9

Neural proliferation, differentiation and control, 1

Neuromedin B

Neuronatin

NIMA (never in mitosis gene a)-related kinase 2

N-methylpurine-DNA glycosylase

Nuclear factor related to kappa B binding protein

Nuclear protein, marker for differentiated aortic smooth muscle and downregulated with vascular injury

Nuclear receptor binding protein

Nucleosome assembly protein 1-like 4

Nucleotide binding protein 2 (MinD homolog, Escherichia coli)

Nudix (nucleoside diphosphate linked moiety X)-type motif 3

NY-REN-24 antigen

Oligodendrocyte lineage transcription factor 2

Opa-interacting protein 5

Origin recognition complex, subunit 6 homolog-like (yeast)

Ornithine decarboxylase antizyme 2

Papillomavirus L2 interacting nuclear protein 1

Paraneoplastic antigen MA2

PDGFA associated protein 1

PDZ and LIM domain 2 (mystique)

Peptidyl prolyl isomerase $\mathrm{H}$ (cyclophilin $\mathrm{H}$ )

Peptidylprolyl isomerase E (cyclophilin E)

Pericentrin 2 (kendrin)

Peripheral myelin protein 2

Peroxisome biogenesis factor 10

Phorbol-12-myristate-13-acetate-induced protein 1

Phosphatidylinositol-4-phosphate 5-kinase, type II, beta

Phosphodiesterase 1C, calmodulin-dependent 70kDa

Phosphofructokinase, liver

Phosphoinositide-3-kinase, regulatory subunit, polypeptide 2 (p85 beta)

Phospholipase D3

Pituitary tumor-transforming 1

Plasminogen activator, tissue

Platelet-activating factor acetylhydrolase $2,40 \mathrm{kDa}$

Platelet-activating factor acetylhydrolase, isoform $\mathrm{Ib}$, gamma subunit $29 \mathrm{kDa}$

Platelet-derived growth factor beta polypeptide (simian sarcoma viral (v-sis) oncogene homolog)

Polycystic kidney disease 1 (autosomal dominant)

Polymerase (DNA directed), delta 2 , regulatory subunit $50 \mathrm{kDa}$

Polymerase (DNA directed), delta 3

Polymerase (DNA directed), epsilon 2 (p59 subunit)

Polymerase (DNA-directed), alpha (70kD)

Polymerase (RNA) II (DNA directed) polypeptide J, $13.3 \mathrm{kDa}$

Presenilin enhancer 2

Prion protein interacting protein

PRKR interacting protein 1 (IL11 inducible)

Programmed cell death 11

Prostaglandin E synthase 2

Protease, serine, 15

Proteasome (prosome, macropain) 26S subunit, ATPase, 3

Proteasome (prosome, macropain) 26S subunit, non-ATPase, 3 
Table 3. Transcripts Common to Human Neural Stem Cells and hes-RPE-TD (Cont'd)

Protein kinase $\mathrm{C}$, mu

Protein phosphatase 1, catalytic subunit, alpha isoform

Protein phosphatase 1, regulatory (inhibitor) subunit 14B

Protein phosphatase 1, regulatory (inhibitor) subunit 15A

Protein phosphatase 1, regulatory (inhibitor) subunit 3C

Protein phosphatase 1, regulatory (inhibitor) subunit 8

Protein phosphatase 1, regulatory subunit 10

Protein phosphatase 1G (formerly 2C), magnesium-dependent, gamma isoform

Protein phosphatase 2 (formerly 2A), regulatory subunit A (PR 65), alpha isoform

Protein phosphatase 4 (formerly $\mathrm{X}$ ), catalytic subunit

Protein regulator of cytokinesis 1

Protein transport protein SEC61 alpha subunit isoform 1

Protein tyrosine phosphatase, nonreceptor type 9

Pseudoautosomal GTP-binding protein-like

Putative G-protein coupled receptor GPCR41

$\mathrm{RAB}$, member of RAS oncogene family-like $2 \mathrm{~A}$

RAB11B, member RAS oncogene family

RAB31, member RAS oncogene family

RAB40B, member RAS oncogene family

RAD51-interacting protein

Radical-fringe homolog (Drosophila)

RAN binding protein 1

Ras and Rab interactor 1

ras Homolog gene family, member $\mathrm{C}$

ras Homolog gene family, member T2

Receptor (calcitonin) activity modifying protein 1

Regulator of G-protein signaling 12

Regulator of G-protein signaling 16

Regulator of G-protein signaling 17

Regulator of G-protein signaling 20

Regulator of nonsense transcripts 1

Regulatory factor $\mathrm{X}$-associated ankyrin-containing protein

Renal tumor antigen

Replication factor C (activator 1) 2, 40kDa

Ribonucleotide reductase M2 polypeptide

Ring finger protein 121

Ring finger protein 126

RNA-binding protein (autoantigenic, hnRNP-associated with lethal yellow)

SATB family member 2

Scavenger receptor class A, member 3

Sentrin/SUMO-specific protease 3

Septin 6

Septin 8

Serine hydroxymethyltransferase 2 (mitochondrial)

Serine/threonine kinase 17a (apoptosis-inducing)

Serine/threonine kinase 18

Serine/threonine kinase 25 (STE20 homolog, yeast)

Serine/threonine kinase 6

SET and MYND domain containing 2

SHC (Src homology 2 domain containing) transforming protein 1

Similar to rat tricarboxylate carrier-like protein

Small nuclear ribonucleoprotein polypeptide C

Smcx Homolog, X-linked (mouse)

Sno, Strawberry notch homolog 1 (Drosophila)

Solute carrier family 12 (potassium/chloride transporters), member 9

Solute carrier family 2 (facilitated glucose transporter), member 3

Solute carrier family 25 (mitochondrial carrier: glutamate), member 22

Solute carrier family 25 (mitochondrial carrier; citrate transporter), member 1

Sorting nexin 1

Sorting nexin 11

Sparc/Osteonectin, cwcv, and kazal-like domains proteoglycan (testican) 2

Spermatogenesis associated 6

Splicing factor $3 \mathrm{~b}$, subunit $4,49 \mathrm{kDa}$ 
Table 3. Transcripts Common to Human Neural Stem Cells and hes-RPE-TD (Cont’o)

Splicing factor, arginine/serine-rich 4

Splicing factor, arginine/serine-rich 8 (suppressor-of-white-apricot homolog, Drosophila)

Sprouty homolog 2 (Drosophila)

Sterile alpha motif domain containing 4

Steroid-5-alpha-reductase, alpha polypeptide 1 (3-oxo-5 alpha-steroid delta 4-dehydrogenase alpha 1)

Stimulated by retinoic acid 13

Stomatin (EPB72)-like 1

Stress-induced-phosphoprotein 1 (Hsp70/Hsp90-organizing protein)

Superkiller viralicidic activity 2-like (S. cerevisiae)

SWI/SNF related, matrix associated, actin dependent regulator of chromatin, subfamily d, member 3

Synaptic vesicle glycoprotein $2 \mathrm{~A}$

Synaptojanin 2

Synaptotagmin I

Syntaxin 10

T54 protein

TAF6 RNA polymerase II, TATA box binding protein (TBP)-associated factor, 80kDa

TAL1 (SCL) interrupting locus

Tenascin C (hexabrachion)

Testis expressed gene 261

Tetracycline transporter-like protein

Thyroid hormone receptor interactor 13

Tissue-factor pathway inhibitor (lipoprotein-associated coagulation inhibitor)

T-LAK cell-originated protein kinase

TNF receptor-associated factor 4

Topoisomerase (DNA) II alpha 170kDa

Transcription factor-like 1

Transcription factor-like 4

Transcription termination factor, RNA polymerase I

Transforming growth factor beta 1 induced transcript 1

Transgelin 2

Transient receptor potential cation channel, subfamily C, member 4 associated protein

Translocase of inner mitochondrial membrane 22 homolog (yeast)

Translocase of inner mitochondrial membrane 44 homolog (yeast)

TRIAD3 protein

TTK protein kinase

Tubulin-specific chaperone c

Tumor necrosis factor receptor superfamily, member $6 \mathrm{~b}$, decoy

Tumor protein D52-like 2

Tumor protein p53 inducible protein 3

Tyrosylprotein sulfotransferase 1

U5 snRNP-specific $40 \mathrm{kDa}$ protein (hPrp8-binding)

UDP-Gal: betaGlcNAc beta 1,4-galactosyltransferase, polypeptide 2

Unc-84 Homolog B (C. elegans)

Uracil-DNA glycosylase

v-akt Murine thymoma viral oncogene homolog 1

Vinexin beta (SH3-containing adaptor molecule-1)

v-jun Sarcoma virus 17 oncogene homolog (avian)

WAS protein family, member 1

WD repeat endosomal protein

Zinc finger protein 143 (clone $\mathrm{pHZ}-1$ )

Zinc finger protein 305

Zinc finger, BED domain containing 4

ZW10 homolog, centromere/kinetochore protein (Drosophila)

Zyxin

Zyxin

NOTE: Comparison of transdifferentiated hES-RPE (hES-RPE-TD) neural precursor microarray data to other data sets demonstrated the similarity of hES-RPE-TD to human neural stem cells (hNSC). After filtering out the genes present in hES-RPE, 437 genes found when our hES-RPE-TD data set was linked to NSC. 
cells faster (3-4 weeks versus 4-8 weeks). RPE differentiation appears to be an inevitable event, provided the cells are given sufficient time; even though less than $1 \%$ of all EBs showed pigmented cells in 4-8 weeks, over the course of 6-9 months all EBs studied became pigmented and, although RPE-like sheets on their surface seemed quiescent (no further EB growth was observed), when plated on suitable substrate they began to rapidly proliferate and were used to establish passageable RPE cultures.

In our system, RPE-like differentiation occurred independently of the presence of serum. RPE cells reliably appeared in cultures grown in the presence or absence of FBS without significant variations in RPE number or time of appearance. The independence of this differentiation pathway on either coculture or extracellular matrix suggests the involvement of other differentiation cues, such as potential autocrine factors produced by differentiating hES cells.

The expression of RPE-specific proteins in these cells correlated with their differentiation states and was similar to what has been previously described for cultured primary RPE. Thus, RPE65 protein and CRALBP were reported to be absent from dedifferentiated human RPE cells (Alge et al., 2003), and our experiments confirmed significantly lower RPE65 mRNA levels in "immature" RPE cultures. CRALBP was present in pigmented epithelial islands and undetectable in "immature" cells, even in established RPE monolayers. Similarly, bestrophin, whose localization in RPE-like monolayers paralleled CRALBP, has been previously identified as a late marker of RPE differentiation, subject to translational control (Bakall et al., 2003). These results confirm further the similarity of hES-derived RPE-like cells to RPE from natural sources at the level of protein expression. Transcriptional profiling shows that hES-RPE is more similar to human fetal RPE than other existing RPE cell lines. Importantly, one of these lines (ARPE-19) has been used successfully in animal transplantation studies to attenuate the loss of visual function (Lund et al., 2001) suggesting that hES-RPE could be a valuable source of tissue for regenerative medicine.

\section{CONCLUSION}

In conclusion, this is the first report of the isolation and characterization of putative RPE cells from human ES cells, as well as the first application of transcriptomics to assess ES cell derivatives and their in vivo counterparts-a "differentiomics" outlook. ARMD alone affects more than 30 million people worldwide and is the leading cause of blindness in patients over 60 in the United States. A significant next step will be to test the ability of these cells to treat this and other retinal degenerative diseases in both humans and animal models. The use of multiple hES-RPE lines in these studies will allow further correlation between function and gene expression. Differentiomics could also be a valuable predictive tool for quality assessment of other ES cell derivatives based on their molecular signature.

\section{ACKNOWLEDGMENTS}

This study was initiated with the support of the Howard Hughes Medical Institution in the laboratory of Dr. Douglas Melton, Harvard University, Boston, MA. The antibody to CRALBP was a generous gift of Dr. John Saari from University of Washington (Seattle, WA). IK would like to thank Dr. Melton for his guidance and encouragement and Jill McMahon for her invaluable help and support during the early stages of this work.

\section{REFERENCES}

Alge, C.S., Suppmann, S., Priglinger, S.G., et al. (2003). Comparative proteome analysis of native differentiated and cultured dedifferentiated human RPE cells. Invest. Ophthalmol. Vis. Sci. 44, 3629-3641.

Abeyta, M.J., Clark, A.T., Rodriguez, R.T., et al. (2004). Unique gene expression signatures of independentlyderived human embryonic stem cell lines. Hum. Mol. Genet. 13, 601-608.

Ashery-Padan, R., and Gruss, P. (2001). Pax6 lights-up the way for eye development [review]. Curr. Opin. Cell Biol. 13, 706-714.

Bakall, B., Marmorstein, L.Y., Hoppe, G., et al. (2003). Expression and localization of bestrophin during normal mouse development. Invest. Ophthalmol. Vis. Sci. 44, 3622-3628.

Baumer, N., Marquardt, T., Stoykova, A., et al. (2003). Retinal pigmented epithelium determination requires the redundant activities of Pax2 and Pax6. Development 130, 2903-2915.

Bunt-Milam, A.H., and Saari, J.C. (1983). Immunocytochemical localization of two retinoid-binding proteins in vertebrate retina. J. Cell Biol. 97, 703-712.

Carpenter, M.K., Inokuma, M.S., Denham, J., et al. (2001). 
Enrichment of neurons and neural precursors from human embryonic stem cells. Exp. Neurol. 172, 383-397.

Chen, S., Samuel, W., Fariss, R.N., et al. (2003). Differentiation of human retinal pigment epithelial cells into neuronal phenotype by $\mathrm{N}$-(4-hydroxyphenyl)retinamide. J. Neurochem. 84, 972-981.

Coffey, P.J., Girman, S., Wang, S.M., et al. (2002). Longterm preservation of cortically dependent visual function in RCS rats by transplantation. Nat. Neurosci. 5, 53-56.

Cowan, C.A., Klimanskaya, I.V., McMahon, J., et al. (2004). Derivation of embryonic stem cell lines from human blastocysts. N. Eng. J. Med. 350, 1353-1356.

Eguchi, G. (2002). Instability in cell commitment of vertebrate pigmented epithelial cells and their transdifferentiation into lens cells [review]. Curr. Top. Dev. Biol. 20, 21-37.

Fischer, A.J., and Reh, T.A. (2000). Identification of a proliferating marginal zone of retinal progenitors in postnatal chickens. Dev. Biol. 220, 197-210.

Fischer, A.J., and Reh, T.A. (2001). Transdifferentiation of pigmented epithelial cells: a source of retinal stem cells? Dev Neurosci. 23(4-5), 268-276.

Grierson, I., Hiscott, P., Hogg, P., et al. (1994). Development, repair and regeneration of the retinal pigment epithelium [review]. Eye 8(Pt 2), 255-262.

Grisanti, S., and Guidry, C. (1995). Transdifferentiation of retinal pigment epithelial cells from epithelial to mesenchymal phenotype. Invest. Ophthalmol. Vis. Sci. 36, 391-405.

Haruta, M., Sasai, Y., Kawasaki, H., et al. (2004). In vitro and in vivo characterization of pigment epithelial cells differentiated from primate embryonic stem cells. Invest. Ophthalmol. Vis. Sci. 45, 1020-1025.

Hirano, M., Yamamoto, A., Yoshimura, N., et al. (2003). Generation of structures formed by lens and retinal cells differentiating from embryonic stem cells. Dev. Dyn. 228, 664-671.

Ivanova, N.B., Dimos, J.T., Schaniel, C., et al. (2002). A stem cell molecular signature. Science 298, 601-604.

Jablonski, M.M., Tombran-Tink, J., Mrazek, D.A., and Iannaccone, A. (2000). Pigment epithelium-derived factor supports normal development of photoreceptor neurons and opsin expression after retinal pigment epithelium removal. J. Neurosci. 20, 7149-7157.

Karakousis, P.C., John, S.K., Behling, K.C., et al. (2001). Localization of pigment epithelium derived factor (PEDF) in developing and adult human ocular tissues. Mol. Vis. 7, 154-163.

Kawasaki, H., Suemori, H., Mizuseki, K., et al. (2002). Generation of dopaminergic neurons and pigmented epithelia from primate ES cells by stromal cell-derived inducing activity. Proc. Natl. Acad. Sci. U.S.A. 99, 1580-1585.

Kennedy, C.J., Rakoczy, P.E., and Constable, I.J. (1996). A simple flow cytometric technique to quantify rod outer segment phagocytosis in cultured retinal pigment epithelial cells. Curr. Eye Res. 15, 998-1003.

Kirchhof, B., Kirchhof, E., Ryan, S.J., and Sorgente, N. (2002). Human retinal pigment epithelial cell cultures: phenotypic modulation by vitreous and macrophages. Exp. Eye Res. 47, 457-463.

Klimanskaya, I., and McMahon, J. (2004). Approaches for derivation and maintenance of human ES cells: detailed procedures and alternatives. In Handbook of Stem Cells, Vol. 1: Embryonic Stem Cells. Lanza R., Gearhart, J., Hogan, B., Melton, D., Pederson, R., Thomson, J., (Elsevier/Academic Press) 437-449.

Laemmli, U.K. (1970). Cleavage of structural proteins during the assembly of the head of bacteriophage T4. Nature 227, 680-685.

Lee, S.C., Kwon, O.W., Seong, G.J., et al. (2001). Epitheliomesenchymal transdifferentiation of cultured RPE cells. Ophthalmic Res. 33, 80-86.

Lund, R.D., Adamson, P., Sauve, Y., et al. (2001). Subretinal transplantation of genetically modified human cell lines attenuates loss of visual function in dystrophic rats. Proc. Natl. Acad. Sci. U.S.A. 98, 9942-9947.

Ma, J., Zhang, J., Othersen, K.L., et al. (2001). Expression, purification, and MALDI analysis of RPE65. Invest. Ophthalmol. Vis. Sci. 42, 1429-1435.

Marmorstein, A.D., Finnemann, S.C., Bonilha, V.L., and Rodriguez-Boulan, E. (1998). Morphogenesis of the retinal pigment epithelium: toward understanding retinal degenerative diseases [review]. Ann. N. Y. Acad. Sci. 857, 1-12.

Marmorstein, A.D., Marmorstein, L.Y., Rayborn, M., et al. (2000). Bestrophin, the product of the Best vitelliform macular dystrophy gene (VMD2), localizes to the basolateral plasma membrane of the retinal pigment epithelium. Proc. Natl. Acad. Sci. U.S.A. 97, 1275812763.

Opas, M., and Dziak, E. (1994). bFGF-induced transdifferentiation of RPE to neuronal progenitors is regulated by the mechanical properties of the substratum. Dev. Biol. 161, 440-454.

Opas, M., Davies, J.R., Zhou, Y., and Dziak, E. (2001). Formation of retinal pigment epithelium in vitro by transdifferentiation of neural retina cells. Int. J. Dev. Biol. 45, 633-642.

Ramalho-Santos, M., Yoon, S., Matsuzaki, Y., et al. (2002). "Stemness": transcriptional profiling of embryonic and adult stem cells. Science 298, 597-600.

Redmond, T.M., Yu, S., Lee, E., et al. (1998). Rpe65 is necessary for production of 11-cis-vitamin A in the retinal visual cycle. Nat. Genet. 20, 344-351.

Reh, T.A., Nagy, T., and Gretton, H. (1987). Retinal pigmented epithelial cells induced to transdifferentiate to neurons by laminin. Nature 330, 68-71.

Rogojina, A.T., Orr, W.E., Song, B.K., and Geisert, E.E., Jr. (2003). Comparing the use of Affymetrix to spotted oligonucleotide microarrays using two retinal pigment epithelium cell lines. Mol. Vis. 9, 482-496.

Sato, N., Sanjuan, I.M., Heke, M., et al. (2003). Molecular signature of human embryonic stem cells and its comparison with the mouse. Dev. Biol 260, 404-413.

Sakaguchi, D.S., Janick, L.M., and Reh, T.A. (1997). Basic fibroblast growth factor (FGF-2) induced transdifferentiation of retinal pigment epithelium: generation of retinal neurons and glia. Dev. Dyn. 209, 387-398. 
Thomson, J.A., Itskovitz-Eldor, J., Shapiro, S.S., et al. (1998). Embryonic stem cell lines derived from human blastocysts. Science 282, 1145-1147; erratum in Science 1998; 282, 1827.

Vinores, S.A., Derevjanik, N.L., Mahlow, J., et al. (1995). Class III beta-tubulin in human retinal pigment epithelial cells in culture and in epiretinal membranes. Exp. Eye Res. 60, 385-400.

Wright, J.M., Zeitlin, P.L., Cebotaru, L., et al. (2004). Gene expression profile analysis of 4-phenylbutyrate treatment of IB3-1 bronchial epithelial cell line demonstrates a major influence on heat-shock proteins. Physiol. Genomics 16, 204-211.

Wright, L.S., Li, J., Caldwell, M.A., Wallace, K., Johnson, J.A., Svenden, C.N. (2003). Gene expression in human neutral stem cells: effects of leukemia inhibitory factor. J Neurochem. 86, 179-195.

Ying, Q.L., Stavridis, M., Griffiths, D., et al. (2003). Conversion of embryonic stem cells into neuroectodermal precursors in adherent monoculture. Nat. Biotechnol. 21, 183-186.

Zhao, S., Rizzolo, L.J., and Barnstable, C.J. (1997). Differentiation and transdifferentiation of the retinal pigment epithelium [review]. Int. Rev. Cytol. 171, 225-266.

Zhao, S., Thornquist, S.C., and Barnstable, C.J. (1995). In vitro transdifferentiation of embryonic rat retinal pigment epithelium to neural retina. Brain Res. 677, 300-310.

Zhao, X., Liu, J., and Ahmad, I. (2002). Differentiation of embryonic stem cells into retinal neurons. Biochem. Biophys. Res. Commun. 297, 177-184.

Address reprint requests to: Dr. Robert Lanza Advanced Cell Technology One Innovation Drive Worcester, MA 01605

E-mail:rlanza@advancedcell.com 